ESAIM: COCV 23 (2017) 195-215

DOI: $10.1051 / \mathrm{cocv} / 2015045$
ESAIM: Control, Optimisation and Calculus of Variations

www.esaim-cocv.org

\title{
A FREE BOUNDARY PROBLEM FOR THE STOKES EQUATIONS
}

\author{
François Bouchon ${ }^{1,2}$, Gunther H. Peichl ${ }^{3, *}$, Mohamed Sayeh $^{4}$ \\ AND RACHID TOUZANI ${ }^{1,2}$
}

\begin{abstract}
A free boundary problem for the Stokes equations governing a viscous flow with overdetermined condition on the free boundary is investigated. This free boundary problem is transformed into a shape optimization one which consists in minimizing a Kohn-Vogelius energy cost functional. Existence of the material derivatives of the states is proven and the corresponding variational problems are derived. Existence of the shape derivative of the cost functional is also proven and the analytic expression of the shape derivative is given in the Hadamard structure form.
\end{abstract}

Mathematics Subject Classification. 35R35, 49Q10, 35Q30, 76D07.

Received November 10, 2014. Revised August 26, 2015. Accepted September 17, 2015.

\section{INTRODUCTION}

This paper aims at studying a free boundary problem for fluid flows governed by the Stokes equations. This problem originates from numerous applications such as magnetic shaping processes, where the shape of the fluid is determined by the Lorentz force. For such configurations, the model is described by the fluid flow equations, here the Stokes equations, and a pressure balance equation on the unknown boundary in the case where surface tension effects are neglected. Depending on the application, two types of models can be considered: an interior problem where the fluid is confined in a mould and has an internal unknown boundary and an exterior problem where a part of the fluid boundary adheres to a solid and the remaining (unknown) part is free and is in contact with the ambient air. We shall focus our study on this last case for two-dimensional geometries.

Consider a bounded $C^{2,1}$ domain $A \subset \mathbb{R}^{2}$ with boundary $\Gamma_{f}$. The fluid is considered in levitation around $A$ and occupies then the domain $\Omega=B \backslash \bar{A}$, where $B$ is a bounded domain with boundary $\Gamma$ that contains $\bar{A}$. Let $\mathbf{u}$ and $p$ stand for the fluid velocity and pressure respectively. Let $\mathbf{f} \in H_{\text {loc }}^{1}\left(\mathbb{R}^{2}\right)^{2}$ denote the density of a given body force and $\mathbf{g} \in H^{\frac{5}{2}}\left(\Gamma_{f}\right)^{2}$ such that

$$
\int_{\Gamma_{f}} \mathbf{g} \cdot \nu=0
$$

where $\nu$ is the outer unit normal vector to $\Gamma_{f}$.

\footnotetext{
Keywords and phrases. Shape derivative, free boundary problems, Stokes Problem.

1 Clermont Université, Université Blaise-Pascal, Laboratoire de Mathématiques, BP 10448, 63000 Clermont-Ferrand, France.

2 CNRS, UMR 6620, LM, 63171 Aubière, France. francois.bouchon, rachid.touzani@univ-bpclermont.fr

3 University of Graz, Institute for Mathematics and Scientific Computing, NAWI Graz, Heinrichstr. 36, 8010 Graz, Austria. gunther.peichl@uni-graz.at

4 Laboratoire de Modélisation Mathématique et Numérique dans les Sciences de l'Ingénieur (LAMSIN), El Manar University, Tunis, Tunisia. Mohamed.Sayeh@ipein.rnu.tn

* The work of this author was supported by the Austrian Science Fund (FWF) under grant SFB F32.
} 
For a given scalar $\lambda$ we consider the free boundary problem of determining the domain $\Omega$ occupied by the fluid, the fluid velocity $\mathbf{u}$ and its pressure $p$ such that:

$$
\begin{cases}-2 \operatorname{div} \sigma(\mathbf{u})+\nabla p=\mathbf{f} & \text { in } \Omega, \\ \operatorname{div} \mathbf{u}=0 & \text { in } \Omega, \\ \mathbf{u}=\mathbf{g} & \text { on } \Gamma_{f}, \\ \mathbf{u} \cdot \nu=0 & \text { on } \Gamma, \\ \nu^{T} \sigma(\mathbf{u}) \tau=0 & \text { on } \Gamma, \\ 2 \nu^{T} \sigma(\mathbf{u}) \nu-p=\lambda & \text { on } \Gamma .\end{cases}
$$

We start with the following observation concerning the scalar $\lambda$ : if $(\Omega, \mathbf{u}, p)$ is a solution of (1.1) corresponding to $\lambda=0$, then $(\Omega, \mathbf{u}, p-\lambda)$ is a solution of the same problem where 0 would be replaced by $\lambda$. For technical reasons, we will need to assume that $\int_{\Omega} p=0$ by adding a constant to the pressure, therefore we need to keep the scalar $\lambda$ in our analysis.

Above, $\nu$ and $\tau$ are the outer normal and tangent unit vectors to the boundary $\Gamma$ respectively and

$$
\sigma(\mathbf{u})=\frac{1}{2}\left(\nabla \mathbf{u}+\nabla \mathbf{u}^{T}\right)
$$

is the symmetric deformation tensor. We note that the prescribed velocity $\mathrm{g}$ expresses the motion of the 'mould' $\Gamma_{f}$.

Problem (1.1) can be viewed as an analog of the Bernoulli free boundary problem, where the Laplace operator is replaced by the Stokes operator. Assuming the existence of a solution to (1.1) we adopt the so-called KohnVogelius formulation, which applies for inverse problems (see $[13,14,26]$ for instance) and which consists in matching a Dirichlet and a Neumann problem. For an application of this technique to the Bernoulli free boundary problem we refer to $[3,9]$.

Hence let $\left(\mathbf{u}_{D}, p_{D}\right),\left(\mathbf{u}_{N}, p_{N}\right)$ denote for given $\Omega$ the solution of the following Dirichlet, respectively Neumann problem:

$$
\begin{cases}-2 \operatorname{div} \sigma\left(\mathbf{u}_{D}\right)+\nabla p_{D}=\mathbf{f} & \text { in } \Omega, \\ \operatorname{div} \mathbf{u}_{D}=0 & \text { in } \Omega, \\ \mathbf{u}_{D}=\mathbf{g} & \text { on } \Gamma_{f}, \\ \nu^{T} \sigma\left(\mathbf{u}_{D}\right) \tau=0 & \text { on } \Gamma, \\ \mathbf{u}_{D} \cdot \nu=0 & \text { on } \Gamma,\end{cases}
$$

and

$$
\begin{cases}-2 \operatorname{div} \sigma\left(\mathbf{u}_{N}\right)+\nabla p_{N}=\mathbf{f} & \text { in } \Omega, \\ \operatorname{div} \mathbf{u}_{N}=0 & \text { in } \Omega, \\ \mathbf{u}_{N}=\mathbf{g} & \text { on } \Gamma_{f}, \\ \nu^{T} \sigma\left(\mathbf{u}_{N}\right) \tau=0 & \text { on } \Gamma, \\ 2 \nu^{T} \sigma\left(\mathbf{u}_{N}\right) \nu-p_{N}=\lambda & \text { on } \Gamma .\end{cases}
$$

Let us define the functional $J$ on a suitable class of domains $\Omega$ by

$$
J(\Omega)=2 \int_{\Omega}\left|\sigma\left(\mathbf{u}_{D}-\mathbf{u}_{N}\right)\right|^{2},
$$

where

$$
|\sigma(\mathbf{u})|^{2}:=\sum_{i, j=1}^{2} \sigma(\mathbf{u})_{i j}^{2}
$$


We observe that $J(\Omega)=0$ if $(\mathbf{u}, \Omega)$ is a solution of the free boundary problem (1.1) since then $\mathbf{u}=\mathbf{u}_{D}=\mathbf{u}_{N}$. Conversely, if $J(\Omega)=0$ Korn's inequality implies $\mathbf{u}_{D}=\mathbf{u}_{N}$. Then $(\mathbf{u}, \Omega)$ with $\mathbf{u}=\mathbf{u}_{D}=\mathbf{u}_{N}$ is a solution to the free boundary problem. Thus Problem (1.1) is equivalent to the shape optimization problem:

$$
\text { Find } \Omega \text { such that } J(\Omega)=\min _{\tilde{\Omega}} J(\tilde{\Omega})=0 \text {. }
$$

Remark 1.1. Clearly the identity $J(\Omega)=0$ is equivalent to the existence of a constant $\alpha \in \mathbb{R}$ such that

$$
\left(\mathbf{u}_{D}, p_{D}\right)=\left(\mathbf{u}_{N}, p_{N}+\alpha\right) .
$$

In this paper we focus on a sensitivity analysis of the cost functional $J$. The computation of the shape gradient is complicated by the fact that applying the method of mapping to perturbations of Problem (1.2) neither preserves the divergence condition $\operatorname{div} \mathbf{u}_{D}=0$ nor the boundary condition $\mathbf{u}_{D} \cdot \nu=0$ on $\Gamma$. We address this issue and propose a novel strategy to overcome the ensuing difficulties in Section 4.2. This technique only needs the weak form of the material derivative of the states.

The slip boundary condition $\mathbf{u} \cdot \nu=0$ on $\Gamma$ allows tangential velocities on the free boundary, but inflow or outflow are not possible. This condition is appropriate for problems that involve free boundaries, flow past chemically reacting walls, and other examples where the usual no-slip condition $\mathbf{u}=0$ is not valid. There has been much discussion regarding the difference between the no-slip and slip boundary conditions. The noslip condition is well established (see, e.g. Girault and Raviart [11] or Galdi [10]) for moderate pressures and velocities by direct observations and comparisons between numerical simulations and experimental results of a large class of complex flow problems. However, early experiments demonstrated that gases at low temperatures slip past solid surfaces. In particular, for sufficiently large Knudsen numbers velocity slip occurs at the wall surface. Such a wall slip has also been observed in the flow of nonlinear fluids such as lubricants, hydraulic fracturing fluids, biological fluids. A slip boundary condition is the appropriate physical model for a wide variety of applications including flow problems with free boundaries such as the coating flow (see $[18,20,22,25])$ and flows past chemically reacting walls (see [4]).

Free boundary problems and inverse problems with similar boundary conditions on the free boundary have already been investigated by several authors (see $[2,16]$ ). Solonnikov [24] considered the problem of filling a plane capillary. The problem of filling a circular capillary tube, coating flows and the steady flow in a capillary tube which is partially filled with liquid were considered. The latter free boundary problem was first considered by Sattinger [19] and investigated in more detail by Solonnikov [23]. As opposed to the problem discussed in this paper the free boundary however is in contact with a rigid wall.

As in most of the cited papers, we consider the simplified model where the boundary conditions on the free boundary do not take into account the surface tension terms. Thus, technical difficulties arising from the higher derivative terms are ignored.

The remainder of this paper is organized as follows: in Section 2 we recall some basic concepts, and results related to shape differentiation are given in Section 3. In Section 4 we prove the existence of the Eulerian derivatives of the state functions $\mathbf{u}_{D}$ and $\mathbf{u}_{N}$ and give the variational formulas verified by them. In Section 5 we prove the existence of the shape derivative of the functional $\mathrm{J}$, and give its analytical representation together with the proof of this expression.

The results presented in Section 3 require the $C^{2,1}$ regularity of the boundary $\Gamma$. Therefore, although many lemmas and theorems of other sections may required weaker assumptions, we consider $\Omega$ (and then $A$ and $B$ ) with $C^{2,1}$ boundaries. Both domains $A$ and $B$ are assumed simply connected.

Remark 1.2. The results presented in this paper can be extended to the 3 -dimensional case. This would need to rewrite some of the technical tools in Section 3, since we use the tangential vector $\tau$ which is defined only in the 2-dimensional situation. 


\section{Discussion of The EQUATIOns FOR $\mathbf{u}_{D}$ AND $\mathbf{u}_{N}$}

In the following $L^{2}(\Omega)$ and $H^{k}(\Omega)$ will stand for classical Lebesgue and Sobolev spaces. Moreover, for $k \geq 0$, we shall denote by $\mathbf{H}^{k}(\Omega)$ the space of vector valued functions $H^{k}(\Omega)^{2}$.

To define the variational formulations we shall make use of the following Hilbert spaces:

$$
\begin{aligned}
\mathbf{V} & =\left\{\mathbf{v} \in \mathbf{H}^{1}(\Omega): \mathbf{v}_{\mid \Gamma_{f}}=0\right\}, \\
\mathbf{V}_{0} & =\{\mathbf{v} \in \mathbf{V}: \mathbf{v} \cdot \nu=0 \text { on } \Gamma\}, \\
\mathbf{V}_{0, \Gamma} & =\left\{\mathbf{v} \in \mathbf{H}^{1}(\Omega): \mathbf{v} \cdot \nu=0 \text { on } \Gamma\right\}, \\
Q & =L^{2}(\Omega), \\
Q_{0} & =\left\{q \in Q: \int_{\Omega} q=0\right\},
\end{aligned}
$$

and the bilinear forms $a: \mathbf{V} \times \mathbf{V} \rightarrow \mathbb{R}, b: \mathbf{V} \times Q \rightarrow \mathbb{R}$ defined by

$$
\begin{aligned}
& a(\mathbf{u}, \mathbf{v})=2 \int_{\Omega} \sigma(\mathbf{u}): \sigma(\mathbf{v}), \\
& b(\mathbf{u}, q)=-\int_{\Omega} q \operatorname{div} \mathbf{u} .
\end{aligned}
$$

The Dirichlet problem can then be cast into the variational formulation:

$$
\begin{cases}\text { Find }\left(\mathbf{u}_{D}, p_{D}\right) \in \mathbf{V}_{0, \Gamma} \times Q_{0} \text { such that: } & \\ a\left(\mathbf{u}_{D}, \mathbf{v}\right)+b\left(\mathbf{v}, p_{D}\right)=\int_{\Omega} \mathbf{f} \cdot \mathbf{v} & \forall \mathbf{v} \in \mathbf{V}_{0}, \\ b\left(\mathbf{u}_{D}, q\right)=0 & \forall q \in Q_{0}, \\ \mathbf{u}_{D}=\mathbf{g} & \text { on } \Gamma_{f} .\end{cases}
$$

We remark that the pressure $p_{D}$ is determined by (1.2) only up to an additive constant.

Similarly one can derive a weak formulation of the Neumann problem (1.3):

$$
\begin{cases}\text { Find }\left(\mathbf{u}_{N}, p_{N}\right) \in \mathbf{H}^{1}(\Omega) \times Q \text { such that: } & \\ a\left(\mathbf{u}_{N}, \mathbf{v}\right)+b\left(\mathbf{v}, p_{N}\right)=\int_{\Omega} \mathbf{f} \cdot \mathbf{v}+\lambda \int_{\Gamma} \mathbf{v} \cdot \nu & \forall \mathbf{v} \in \mathbf{V}, \\ b\left(\mathbf{u}_{N}, q\right)=0 & \forall q \in Q \\ \mathbf{u}_{N}=\mathbf{g} & \text { on } \Gamma_{f} .\end{cases}
$$

Existence and uniqueness of the solutions $\left(\mathbf{u}_{N}, p_{N}\right) \in \mathbf{H}^{1}(\Omega) \times Q$ and $\left(\mathbf{u}_{D}, p_{D}\right) \in \mathbf{V}_{0, \Gamma} \times Q_{0}$ to the mixed variational problems (2.1) and (2.2), follow from the following properties satisfied by the bilinear forms $a$ and $b$,

(i) The bilinear form $a$ is coercive, i.e. there is a constant $C>0$ such that

$$
a(\mathbf{v}, \mathbf{v}) \geq C\|\mathbf{v}\|_{\mathbf{H}^{1}(\Omega)}^{2} \quad \forall \mathbf{v} \in \mathbf{V},
$$

(ii) The bilinear form $b$ satisfies the inf-sup condition, i.e. there is a constant $\beta>0$ such that

$$
\inf _{q \in Q, q \neq 0} \sup _{\mathbf{v} \in \mathbf{V}, \mathbf{v} \neq 0} \frac{b(\mathbf{v}, q)}{\|\mathbf{v}\|_{\mathbf{H}^{1}(\Omega)}\|q\|_{L^{2}(\Omega)}}>\beta .
$$


For further reference we recall the following regularity result:

Theorem 2.1 (see Thm. 4.1 in [8]).

Let $\Omega \in \mathbb{R}^{n}$ be a bounded domain of class $C^{k, \ell}$ with $1 \leq k, 0 \leq \ell \leq 1$. There exists a Lipschitz continuous function $f: \mathbb{R}^{n} \rightarrow \mathbb{R}^{n}$ such that:

$$
\Omega=\left\{y \in \mathbb{R}^{n}, f(y)>0\right\}, \partial \Omega=\left\{y \in \mathbb{R}^{n}, f(y)=0\right\}
$$

and a neighborhood $\omega$ of $\partial \Omega$ such that:

$$
f \in C^{k, \ell}(\omega), \nabla f \neq 0 \text { on } \omega \text { and } \nu=-\frac{\nabla f}{|\nabla f|}
$$

where $\nu$ is the outer unit normal to $\Omega$ on $\partial \Omega$.

The verification of the inf-sup condition for (2.2) is based on the following Lemma which is adapted from [6].

Lemma 2.2. Let $\Omega \subset \mathbb{R}^{2}$ be a doubly connected $C^{1,1}$-domain with boundary $\Gamma \cup \Gamma_{f}$. Then there exists a constant $C>0$ such that for all $p \in L^{2}(\Omega)$ there is a function $\mathbf{v} \in \mathbf{H}^{1}(\Omega)$ satisfying

$$
\begin{aligned}
\operatorname{div} \mathbf{v} & =p \quad \text { in } \Omega, \\
\mathbf{v} & =0 \quad \text { on } \Gamma_{f}, \\
\|\mathbf{v}\|_{\mathbf{H}^{1}} & \leq C\|p\|_{L^{2}} .
\end{aligned}
$$

Proof. For given $p \in L^{2}(\Omega)$, consider the problem

$$
\left\{\begin{aligned}
\operatorname{div} \mathbf{v} & =p & & \text { in } \Omega \\
\mathbf{v} & =\mathbf{0} & & \text { on } \Gamma_{f} \\
\mathbf{v} & =K \nu & & \text { on } \Gamma
\end{aligned}\right.
$$

where $K=\frac{1}{|\Gamma|} \int_{\Omega} p$.

The result follows from Lemma 3.3 in [1] observing that $\nu \in C^{0,1}(\omega) \subset W^{1, \infty}(\omega) \subset H^{1}(\omega) \subset H^{1 / 2}(\Gamma)$ by Theorem 2.1.

Using $\mathbf{v} \in \mathbf{V}$ as specified by Lemma 2.2 one can show the inf-sup condition for problem (2.2) similarly as for problem (2.1). Hence problem (2.2) is also well-posed.

We conclude this section with an interpretation of the boundary condition in (2.2) which is a priori not clear since $p_{N} \in L^{2}(\Omega)$ only. We note that the first equation in (2.2) can be written as

$$
2 \int_{\Omega} \sigma(\mathbf{u}): \nabla \mathbf{v}-\int_{\Omega} p \operatorname{div} \mathbf{v}=\int_{\Omega} \mathbf{f} \cdot \mathbf{v}+\lambda \int_{\Gamma} \mathbf{v} \cdot \nu, \quad \mathbf{v} \in \mathbf{V},
$$

which implies that the equation

$$
-2 \operatorname{div} \sigma(\mathbf{u})+\nabla p=\mathbf{f}
$$

is satisfied in the distributional sense. Since

$$
(-2 \operatorname{div} \sigma(\mathbf{u})+\nabla p)_{i}=\operatorname{div}(-2 \sigma(\mathbf{u})+p I) e_{i}, \quad i=1,2,
$$

where $e_{i}$ is the $i$ th vector of the canonical basis in $\mathbb{R}^{2}$ and $(-2 \sigma(\mathbf{u})+p I) e_{i} \in \mathbf{L}^{2}(\Omega)$ we conclude that $(-2 \sigma(\mathbf{u})+$ $p I) e_{i} \in \mathbf{H}^{1}(\operatorname{div}, \Omega), i=1,2$. In view of Theorem 2.5 in [11], the normal component $e_{i}^{T}(-2 \sigma(\mathbf{u})+p I) \nu, i=1,2$, exists in $H^{-\frac{1}{2}}(\Gamma)$ and the following Green's formula holds for $\varphi \in H^{1}(\Omega)$ :

$$
\left((-2 \sigma(\mathbf{u})+p I) e_{i}, \nabla \varphi\right)+\left(\operatorname{div}(-2 \sigma(\mathbf{u})+p I) e_{i}, \varphi\right)=\left\langle e_{i}^{T}(-2 \sigma(\mathbf{u})+p I) \nu, \varphi\right\rangle
$$


$\left(\langle\cdot, \cdot\rangle\right.$ denotes the duality pairing between $H^{\frac{1}{2}}(\Gamma)$ and $\left.H^{-\frac{1}{2}}(\Gamma)\right)$. Since for $\psi \in \mathbf{H}^{1}(\Omega)$ one can verify

$$
\begin{aligned}
\left((-2 \sigma(\mathbf{u})+p I) e_{i}, \nabla \psi_{i}\right) & =-2 \int_{\Omega} \sigma(\mathbf{u}): \sigma(\psi)+\int_{\Omega} p \operatorname{div} \psi, \\
\left(\operatorname{div}(-2 \sigma(\mathbf{u})+p I) e_{i}, \psi_{i}\right) & =(-2 \operatorname{div} \sigma(\mathbf{u})+\nabla p, \psi)
\end{aligned}
$$

we obtain the identity

$$
\int_{\Omega}(-2 \sigma(\mathbf{u}): \sigma(\psi)+p \operatorname{div} \psi)+\int_{\Omega}(-2 \operatorname{div} \sigma(\mathbf{u})+\nabla p) \cdot \psi=\langle(-2 \sigma(\mathbf{u})+p I) \nu, \psi\rangle .
$$

In view of (2.3) and (2.4) this implies

$$
-\lambda \int_{\Gamma} \psi \cdot \nu=\langle(-2 \sigma(\mathbf{u})+p I) \nu, \psi\rangle
$$

Hence $(\mathbf{u}, p) \in \mathbf{H}^{1}(\Omega) \times Q$ satisfies the boundary condition

$$
(2 \sigma(\mathbf{u})-p I) \cdot \nu=\lambda \nu
$$

in $\mathbf{H}^{-\frac{1}{2}}(\Gamma)$. If the solution turns out more regular, e.g. $\left(\mathbf{u}_{N}, p_{N}\right) \in \mathbf{H}^{2}(\Omega) \times H^{1}(\Omega)$ this implies

$$
\begin{aligned}
2 \nu^{T} \sigma\left(\mathbf{u}_{N}\right) \tau=0 & \text { on } \Gamma, \\
2 \nu^{T} \sigma\left(\mathbf{u}_{N}\right) \nu-p=\lambda & \text { on } \Gamma .
\end{aligned}
$$

A similar discussion can be carried out for $\left(\mathbf{u}_{D}, p_{D}\right)$.

In order to derive the shape derivative of $J$ we need $\left(\mathbf{u}_{D}, p_{D}\right) \in \mathbf{H}^{3}(\Omega) \times H^{2}(\Omega)$ which is guaranteed by ([17], Thm. 6.2):

Theorem 2.3. Assume that $\Gamma_{f}$ and $\Gamma$ are of class $C^{3}$ and $C^{4}$ respectively, $\mathbf{f} \in \mathbf{H}^{1}(\Omega), \mathbf{g} \in \mathbf{H}^{\frac{5}{2}}\left(\Gamma_{f}\right)$, then

$$
\left(\mathbf{u}_{D}, p_{D}\right) \in \mathbf{H}^{3}(\Omega) \times H^{2}(\Omega)
$$

Moreover

$$
\left\|\mathbf{u}_{D}\right\|_{3}+\left\|p_{D}\right\|_{2} \leq C\left(\|\mathbf{f}\|_{1}+\|\mathbf{g}\|_{\frac{5}{2}}\right) .
$$

We point out that under the assumptions of Theorem 2.3 we also have $\left(u_{N}, p_{N}\right) \in \mathbf{H}^{3}(\Omega) \times \mathbf{H}^{2}(\Omega)$. Results in [2] indicate that this regularity could be obtained assuming $\Omega \in C^{2,1}$ only (see also [5], Thm. IV.7.4). Given this regularity of the solution one only needs $C^{2,1}$-regularity of $\Omega$ to justify the derivation of the shape derivative of $J$. Therefore we shall assume for the rest of the paper $\Omega \in C^{2,1}$ and $\left(\mathbf{u}_{D}, p_{D}\right),\left(\mathbf{u}_{N}, p_{N}\right) \in \mathbf{H}^{3}(\Omega) \times H^{2}(\Omega)$.

\section{Some MATERIAL ON THE SHAPE DERIVATIVE OF $J$}

In this section we briefly describe the idea of the shape derivative of $J$ and recall some additional results which will be needed in the sequel. For a more thorough exposition we refer to $[8,12,21]$. In order to define the shape derivative of $J$ we embed problems (1.2) and (1.3) into a family of perturbed problems which are defined on perturbations of a $C^{2,1}$ reference domain $\Omega$ constructed by perturbing the identity. Let $U$ be a convex bounded domain of class $C^{2,1}$ such that $\bar{\Omega} \subset U$ and let

$$
\mathcal{S}=\left\{\mathbf{h} \in C^{2,1}\left(\bar{U}, \mathbb{R}^{2}\right): \mathbf{h}=0 \text { on } \partial U \cup \Gamma_{f} \text { and } \mathbf{h} \cdot \tau=0 \text { on } \Gamma\right\}
$$

be the space of feasible deformation fields endowed with the natural norm in $C^{2}\left(\bar{U}, \mathbb{R}^{2}\right)$. This choice of feasible deformation fields anticipates the form of the shape gradient of $J$ according to the Hadamard-Zolésio structure theorem (see $[7,15]$ ). For a fixed field $\mathbf{h} \in \mathcal{S}$ define for every $t \in \mathbb{R}$ the mapping $F_{t}: \bar{U} \rightarrow \mathbb{R}^{2}$ by

$$
F_{t}=i d+t \mathbf{h}
$$


For $t$ sufficiently small $F_{t}$ defines a family of $C^{2}$-diffeomorphisms of $U$ onto itself. For such $t$ one sets

$$
\Omega_{t}=F_{t}(\Omega), \quad \Gamma_{t}=F_{t}(\Gamma)
$$

hence $\Omega_{0}=\Omega, \Gamma_{0}=\Gamma, \Gamma_{f}=F_{t}\left(\Gamma_{f}\right)$ and $\bar{\Omega}_{t} \subset U$. For each $t$ sufficiently small we consider problems $(1.2)_{t}$ and $(1.3)_{t}$ which correspond to problems (1.2) and (1.3) with $\left(\Omega, \Gamma_{f}, \Gamma\right)$ replaced by $\left(\Omega_{t}, \Gamma_{f}, \Gamma_{t}\right)$. In view of the previous discussion $(1.2)_{t}$ and $(1.3)_{t}$ have unique weak solutions $\left(\mathbf{u}_{D, t}, p_{D, t}\right) \in V_{0, \Gamma_{t}} \times Q_{0, t}$, respectively $\left(\mathbf{u}_{N, t}, p_{N, t}\right) \in \mathbf{H}^{1}\left(\Omega_{t}\right) \times Q_{t}$ where

$$
\begin{aligned}
Q_{t} & =L^{2}\left(\Omega_{t}\right), \\
Q_{0, t} & =\left\{q_{t} \in Q_{t}: \int_{\Omega_{t}} q_{t}=0\right\},
\end{aligned}
$$

Set

$$
J\left(\Omega_{t}\right)=2 \int_{\Omega_{t}}\left|\sigma\left(\mathbf{u}_{D, t}-\mathbf{u}_{N, t}\right)\right|^{2} .
$$

Then the Eulerian derivative of $J$ at $\Omega$ in the direction $\mathbf{h}$ is defined as

$$
J^{\prime}(\Omega ; \mathbf{h})=\lim _{t \rightarrow 0} \frac{1}{t}\left(J\left(\Omega_{t}\right)-J(\Omega)\right) .
$$

The functional $J$ is called shape differentiable at $\Omega$ if $J^{\prime}(\Omega ; \mathbf{h})$ exists for all $\mathbf{h} \in \mathcal{S}$ and defines a continuous linear functional on $\mathcal{S}$.

In order to evaluate $J\left(\Omega_{t}\right)-J(\Omega)$ we transform $J\left(\Omega_{t}\right)$ to an integral over the reference domain $\Omega$. For this purpose we refer any function $\varphi_{t}: \Omega_{t} \rightarrow \mathbb{R}^{k}, k=1,2$, to the reference domain using the transformation

$$
\varphi^{t}=\varphi_{t} \circ F_{t}: \Omega \rightarrow \mathbb{R}^{k}
$$

Let us define the material and shape derivatives of a function depending on the domain.

Definition 3.1. Let $u(\Omega)$ be a function defined and depending on the domain $\Omega$. The material derivative of $u$ in the direction $\mathbf{h}$ at $\Omega$ is the limit

$$
\dot{u}(\mathbf{h}):=\lim _{t \rightarrow 0} \frac{u\left(\Omega_{t}\right) \circ F_{t}-u(\Omega)}{t},
$$

when it exists. The local derivative also called shape derivative in the direction $\mathbf{h}$ at $\Omega$ is the limit

$$
u^{\prime}(\mathbf{h}):=\lim _{t \rightarrow 0} \frac{u\left(\Omega_{t}\right)-u(\Omega)}{t} .
$$

The general strategy to derive shape derivatives is to first transfer the problem back to the original boundary before computing the limit, which results in the need to compute the material derivative. The chain rule combines both by the identity

$$
\dot{u}(\mathbf{h})=u^{\prime}(\mathbf{h})+\nabla u \cdot \mathbf{h} .
$$

Thus, if $u$ does not depend on the geometry, one has

$$
u^{\prime}(\mathbf{h})=0 \quad \text { and } \quad \dot{u}(\mathbf{h})=\nabla u \cdot \mathbf{h} .
$$

The following technical results (see [8], Chap. 9) will be useful in deriving equations for the transported solutions $\left(\mathbf{u}_{D}^{t}, p_{D}^{t}\right)$ and $\left(\mathbf{u}_{N}^{t}, p_{N}^{t}\right)$. 
Lemma 3.2. Let $\varphi_{t} \in H^{1}\left(\Omega_{t}\right)$ and $\mathbf{v}_{t} \in \mathbf{H}^{1}\left(\Omega_{t}\right)$. Then $\varphi^{t} \in H^{1}(\Omega), \mathbf{v}^{t} \in \mathbf{H}^{1}(\Omega)$ and we have

$$
\begin{aligned}
& \left(\nabla \varphi_{t}\right) \circ F_{t}=\left(\nabla \varphi_{t}\right)^{t}=M_{t} \nabla \varphi^{t}, \\
& \left(\operatorname{div} \mathbf{v}_{t}\right) \circ F_{t}=\left(\operatorname{div} \mathbf{v}_{t}\right)^{t}=M_{t}^{T}: \nabla \mathbf{v}^{t},
\end{aligned}
$$

where

$$
M_{t}=\left(D F_{t}\right)^{-T}=\left(\nabla F_{t}\right)^{-1} .
$$

Here, $D$ denotes the Jacobian matrix and $\nabla=D^{T}$.

For further use we recall the definition of the tangential divergence of a vector field $\varphi$ and the tangential gradient of a sufficiently smooth function $f$ :

$$
\begin{aligned}
\operatorname{div}_{\Gamma} \varphi & =\operatorname{div} \varphi_{\mid \Gamma}-\nabla \varphi \nu \cdot \nu, \\
\nabla_{\Gamma} f & =\nabla f_{\mid \Gamma}-\frac{\partial f}{\partial \nu} \nu .
\end{aligned}
$$

Next we briefly discuss the variation of the normal field $\nu_{t}$. Let $b_{t}$ denote the signed distance function to $\Gamma_{t}$ such that $\nu_{t}=\nabla b_{t}$. By ([8], Thm. 5.4.3) we have $b_{t} \in C^{2}$ in a neighborhood of $\Gamma_{t}$. Define

$$
b^{t}=b_{t} \circ F_{t}, \quad \nu^{t}=\nu_{t} \circ F_{t} .
$$

Since $\Gamma$ is the zero level set of $b^{t}$ we conclude that $\nabla b^{t}$ is collinear to $\nu=\nu_{0}$ and $\nabla b^{t}=\alpha \nu$ for some $\alpha>0$. This implies

$$
\nu^{t}=\nabla b_{t} \circ F_{t}=M_{t} \nabla b^{t}=\alpha M_{t} \nu .
$$

In view of $\left|\nu^{t}\right|=1$ one eventually obtains

$$
\nu^{t}=\frac{M_{t} \nu}{\left|M_{t} \nu\right|}
$$

Since $\nabla b$ provides an unitary $C^{1,1}$-extension of $\nu$ to a neighborhood of $\Gamma$ we deduce from (3.2) the following properties of the normal and tangent vector fields:

Lemma 3.3. Let $\Omega$ be a $C^{2,1}$ domain. We have:

$$
\begin{aligned}
& (\nabla \nu) \nu=(\nabla \tau) \tau=0 \\
& (\nabla \nu) \tau+(\nabla \tau) \nu=0 \\
& (\nabla \nu) \tau=-(\nabla \tau) \nu=\kappa \tau
\end{aligned}
$$

where $\kappa$ denotes the curvature.

Proof. (3.3) and (3.4) follow by differentiating the identities $|\nu|^{2}=|\tau|^{2}=1$, respectively $\nu \cdot \tau=0$. (3.5) is a consequence of the following identities:

$$
(\nabla \nu) \tau \cdot \nu=0, \quad(\nabla \nu) \tau \cdot \tau=\operatorname{div}_{\Gamma} \nu=\kappa
$$

and (3.4).

The relations (3.3)-(3.5) even hold on a neighborhood of $\Gamma$ since $b$ is defined and regular on a neighborhood of $\Gamma$.

Finally we report some properties of the deformation fields $\mathbf{h} \in \mathcal{S}$. 
Lemma 3.4. Let $\mathbf{h} \in \mathcal{S}$. Then the following relations hold on $\Gamma$ :

$$
\begin{aligned}
& \nabla(\mathbf{h} \cdot \nu)=(\nabla \mathbf{h}) \nu, \\
& \operatorname{div}_{\Gamma} \mathbf{h}=\kappa \mathbf{h} \cdot \nu .
\end{aligned}
$$

Proof. Combining $\mathbf{h}=(\mathbf{h} \cdot \nu) \nu$ on $\Gamma$ and (3.3) we obtain

$$
\nabla(\mathbf{h} \cdot \nu)=(\nabla \mathbf{h}) \nu+(\nabla \nu) \mathbf{h}=(\nabla \mathbf{h}) \nu+(\mathbf{h} \cdot \nu)(\nabla \nu) \nu=(\nabla \mathbf{h}) \nu .
$$

For the proof of (3.7) we observe that $\operatorname{div}_{\Gamma} \mathbf{h}$ does not depend on the extension $\tilde{\mathbf{h}}$ of $\mathbf{h}$ into a neighborhood of $\Gamma$. Since $\mathbf{h}=(\mathbf{h} \cdot \nu) \nu$ on $\Gamma$ one can use the identity

$$
\tilde{\mathbf{h}}=(\mathbf{h} \cdot \nabla b) \nabla b=(\mathbf{h} \cdot \nu) \nu
$$

for the computation of $\operatorname{div}_{\Gamma} \mathbf{h}$. Thus we obtain

$$
\operatorname{div}_{\Gamma} \mathbf{h}=\operatorname{div}_{\Gamma} \tilde{\mathbf{h}}=\operatorname{div} \tilde{\mathbf{h}}-(\nabla \tilde{\mathbf{h}}) \nu \cdot \nu .
$$

Since $\nabla \tilde{\mathbf{h}}=\nabla(\mathbf{h} \cdot \nu) \nu^{T}+(\mathbf{h} \cdot \nu) \nabla \nu,(3.3)$ leads to

$$
(\nabla \tilde{\mathbf{h}}) \nu \cdot \nu=\nabla(\mathbf{h} \cdot \nu) \nu^{T} \nu \cdot \nu+(\mathbf{h} \cdot \nu)(\nabla \nu) \nu \cdot \nu=\nabla(\mathbf{h} \cdot \nu) \cdot \nu .
$$

Using $\operatorname{div} \nu=\kappa$ we therefore find

$$
\begin{aligned}
\operatorname{div}_{\Gamma} \mathbf{h} & =\operatorname{div}((\mathbf{h} \cdot \nu) \nu)-\nabla(\mathbf{h} \cdot \nu) \cdot \nu \\
& =\nabla(\mathbf{h} \cdot \nu) \cdot \nu+(\mathbf{h} \cdot \nu) \operatorname{div} \nu-\nabla(\mathbf{h} \cdot \nu) \cdot \nu=\kappa(\mathbf{h} \cdot \nu) .
\end{aligned}
$$

As a consequence of (3.2) and Lemma 3.4 we obtain the following expression for the material derivative of the normal field (see also [12]).

Lemma 3.5. For each $t$ sufficiently small $\nu^{t}$ is $C^{1}$ in a neighborhood of $\Gamma$ and $t \mapsto \nu^{t}(x)$ is differentiable at $t=0$ uniformly in $x \in \Gamma$ and its derivative at 0 is given by the following expression:

$$
\dot{\nu}=-\nabla_{\Gamma}(\mathbf{h} \cdot \nu) \text {. }
$$

A similar statement holds for the transported tangent vector $\tau^{t}$. This follows from the representation

$$
\tau^{t}=\frac{1}{\left|M_{t} \nu\right|}\left(-\left(M_{t} \nu \cdot \tau\right) \nu+\left(M_{t} \nu \cdot \nu\right) \tau\right)
$$

Lemma 3.6. For $f, g \in H^{2}(\Omega)$ the following integration by parts formula holds

$$
\int_{\partial \Omega} f(\nabla g \cdot \tau)=-\int_{\partial \Omega} g(\nabla f \cdot \tau)
$$

In particular we have for $\mathbf{h} \in \mathcal{S}$

$$
\int_{\Gamma} f \nabla \mathbf{h} \nu \cdot \tau=-\int_{\Gamma}(\nabla f \cdot \tau) \mathbf{h} \cdot \nu
$$

Proof. Recall that the following integration by parts formula holds for domains of class $C^{2}$ and $\varphi \in H^{2}(\Omega)$ and $\psi \in \mathbf{C}^{1}(\bar{\Omega})$ such that $\psi \cdot \nu=0$ on $\partial \Omega$

$$
\int_{\partial \Omega} \nabla_{\Gamma} \varphi \cdot \psi=-\int_{\partial \Omega} \varphi \operatorname{div}_{\Gamma} \psi .
$$


Choose $\varphi=g$ and $\psi=f_{n} \tau$ where $\left(f_{n}\right) \subset C^{1}(\bar{\Omega})$ approximates $f$ in $H^{2}(\Omega)$ and $\tau$ is a continuously differentiable extension of the tangent field to $\Omega$ which is unitary in a neighborhood of $\partial \Omega$. Note that in such a neighborhood of $\partial \Omega$ we have $\tau=\left(-b_{y}, b_{x}\right)$ and hence $\operatorname{div} \tau=0$. This entails

$$
\operatorname{div}_{\Gamma}\left(f_{n} \tau\right)=\nabla f_{n} \cdot \tau-D\left(f_{n} \tau\right) \nu \cdot \nu
$$

Then (3.5) implies

$$
D\left(f_{n} \tau\right) \nu \cdot \nu=D f_{n} \nu \tau \cdot \nu+f_{n} D \tau \nu \cdot \nu=0 .
$$

Since $\nabla g \cdot \tau=\nabla_{\Gamma} g \cdot \tau$ we obtain

$$
\int_{\partial \Omega} f_{n}(\nabla g \cdot \tau)=-\int_{\partial \Omega} g\left(\nabla f_{n} \cdot \tau\right)
$$

which implies the result. The second statement follows choosing $g=\mathbf{h} \cdot \nu$ using (3.6). Note that $g$ vanishes on $\Gamma_{f}$.

We conclude this section with some well known formula:

$$
\left.\frac{\mathrm{d} M_{t}}{\mathrm{~d} t}\right|_{t=0}=-\nabla \mathbf{h} \quad \text { and then }\left.\frac{\mathrm{d} M_{t}^{-T}}{\mathrm{~d} t}\right|_{t=0}=\nabla \mathbf{h}^{T}
$$

and

$$
\left.\frac{\mathrm{d} \delta_{t}}{\mathrm{~d} t}\right|_{t=0}=\operatorname{div} \mathbf{h}
$$

where we have set $\delta_{t}=\operatorname{Det}\left(D F_{t}\right)=\operatorname{det}(I+t D \mathbf{h})$.

\section{Differentiability of the states}

For each $t \in I$ we define the bilinear forms $a^{t}, \dot{a}: \mathbf{V} \times \mathbf{V} \rightarrow \mathbb{R}$ and $b^{t}, \dot{b}: \mathbf{V} \times Q \rightarrow \mathbb{R}$ by

$$
\begin{aligned}
a^{t}(\mathbf{u}, \mathbf{v}) & =\int_{\Omega} \delta_{t} M_{t}^{T}\left(M_{t} \nabla \mathbf{u}+\left(M_{t} \nabla \mathbf{u}\right)^{T}\right): \nabla \mathbf{v} \\
\dot{a}(\mathbf{u}, \mathbf{v}) & =2 \int_{\Omega}(\operatorname{div} h) \sigma(\mathbf{u}): \nabla \mathbf{v}-2 \int_{\Omega} \sigma(\mathbf{u}): \nabla h \nabla \mathbf{v}-2 \int_{\Omega} \nabla h \nabla \mathbf{u}: \sigma(\mathbf{v}) \\
b^{t}(\mathbf{u}, q) & =-\int_{\Omega} \delta_{t} q\left(M_{t}^{T}: \nabla \mathbf{u}\right) \\
\dot{b}(\mathbf{u}, q) & =-\int_{\Omega} q \operatorname{div} h \operatorname{div} \mathbf{u}+\int_{\Omega} q \nabla h^{T}: \nabla \mathbf{u} .
\end{aligned}
$$

\subsection{Differentiability of $t \mapsto\left(\mathrm{u}_{N}^{t}, p_{N}^{t}\right)$}

It was shown in Section 2 that the family of perturbed Neumann problems $(1.3)_{t}$ is well posed in $\mathbf{H}^{1}\left(\Omega_{t}\right) \times Q_{t}$ for $t$ in a sufficiently small neighborhood $I$ of 0 . We observe that the transported solutions $\left(\mathbf{u}_{N}^{t}, p_{N}^{t}\right)$ belong to $\mathbf{V} \times Q$. One can verify that $\left(\mathbf{u}_{N}^{t}, p_{N}^{t}\right)$ satisfies the following system.

Theorem 4.1. For each $t \in I$ the pair $\left(\mathbf{u}_{N}^{t}, p_{N}^{t}\right) \in \mathbf{H}^{1}(\Omega) \times Q$ is the unique solution of the problem

$$
\begin{cases}a^{t}\left(\mathbf{u}_{N}^{t}, \varphi\right)+b^{t}\left(\varphi, p_{N}^{t}\right)=\int_{\Omega} \delta_{t} \mathbf{f}^{t} \cdot \varphi+\lambda \int_{\Gamma} \delta_{t} \varphi \cdot M_{t} \nu & \forall \mathbf{v} \in \mathbf{V}, \\ b^{t}\left(\mathbf{u}_{N}^{t}, \chi\right)=0 & \forall \chi \in Q, \\ \mathbf{u}_{N}^{t}=\mathbf{g} & \text { on } \Gamma_{f} .\end{cases}
$$


Proof. We just indicate the transformation of the boundary integral in $(2.2)_{t}$ describing the Neumann condition. The transformation formula for boundary integrals implies

$$
\int_{\Gamma_{t}} \varphi_{t} \cdot \nu_{t}=\int_{\Gamma} \varphi^{t} \cdot \nu^{t} \delta_{t}\left|M_{t} \nu\right|=\int_{\Gamma} \delta_{t} \varphi^{t} \cdot M_{t} \nu
$$

where we used (3.2) in the last step (see also [2], Lem. 3.1).

Now we can state the main result of this section.

Theorem 4.2. The mapping $t \mapsto\left(\mathbf{u}_{N}^{t}, p_{N}^{t}\right)$ is continuously strongly differentiable on a neighborhood of zero. Furthermore,

$$
\left(\dot{\mathbf{u}}_{N}, \dot{p}_{N}\right):=\left.\frac{\partial}{\partial t}\left(\mathbf{u}_{N}^{t}, p_{N}^{t}\right)\right|_{t=0},
$$

is the unique solution of the following variational problem:

$$
\begin{cases}\text { Find }\left(\dot{\mathbf{u}}_{N}, \dot{p}_{N}\right) \in \mathbf{V} \times Q \text { such that: } & \\ a\left(\dot{\mathbf{u}}_{N}, \varphi\right)+b\left(\varphi, \dot{p}_{N}\right)=L_{N}(\varphi) & \forall \varphi \in \mathbf{V}, \\ b\left(\dot{\mathbf{u}}_{N}, \chi\right)=-\dot{b}\left(\mathbf{u}_{N}, \chi\right) & \forall \chi \in Q,\end{cases}
$$

where $L_{N}(\varphi)$ is given by

$$
L_{N}(\varphi):=-\dot{a}\left(\mathbf{u}_{N}, \varphi\right)-\dot{b}\left(\varphi, p_{N}\right)+\ell_{N}(\varphi)
$$

and

$$
\ell_{N}(\varphi)=\int_{\Omega}\left(\operatorname{div} \mathbf{h} \mathbf{f} \cdot \varphi+\nabla \mathbf{f}^{T} h \cdot \varphi\right)+\lambda \int_{\Gamma}(\operatorname{div} \mathbf{h} \varphi \cdot \nu-\varphi \cdot(\nabla \mathbf{h}) \nu) .
$$

For $\varphi \in \mathbf{V} \cap \mathbf{H}^{2}(\Omega)$ one can represent $\ell_{N}$ as

$$
\ell_{N}(\varphi)=\int_{\Omega}\left(\operatorname{div} \mathbf{h} \mathbf{f} \cdot \varphi+\nabla \mathbf{f}^{T} h \cdot \varphi\right)+\lambda \int_{\Gamma}(\nabla \varphi \tau \cdot \tau) \mathbf{h} \cdot \nu .
$$

Proof. Let us consider the mapping

$$
H=\left(H_{1}, H_{2}\right): I \times \mathbf{V} \times Q \rightarrow \mathbf{V}^{*} \times Q^{*}
$$

defined by

$$
\begin{aligned}
\left\langle H_{1}(t, \mathbf{w}, \theta), \varphi\right\rangle= & a^{t}\left(\mathbf{w}+\mathbf{u}_{N}, \varphi\right)+b^{t}(\varphi, \theta) & & \\
& -\lambda \int_{\Gamma} \delta_{t} \varphi \cdot M_{t} \nu-\int_{\Omega} \delta_{t} \mathbf{f}^{t} \cdot \varphi, & & \varphi \in \mathbf{V} \\
\left\langle H_{2}(t, \mathbf{w}, \theta), \chi\right\rangle= & b^{t}\left(\mathbf{w}+\mathbf{u}_{N}, \chi\right), & & \chi \in Q .
\end{aligned}
$$

Then $\left(\mathbf{u}_{N}^{t}-\mathbf{u}_{N}, p_{N}^{t}\right)$ is the unique element of $\mathbf{V} \times Q$ which satisfies

$$
H\left(t, \mathbf{u}_{N}^{t}-\mathbf{u}_{N}, p_{N}^{t}\right)=0
$$

for each $t \in I$. Since

$$
\left\langle D_{(\mathbf{w}, \theta)} H\left(0, \mathbf{0}, p_{N}\right)(\delta \mathbf{w}, \delta \theta),(\varphi, \chi)\right\rangle=(a(\delta \mathbf{w}, \varphi)+b(\varphi, \delta \theta), b(\delta \mathbf{w}, \chi))
$$


it follows from the discussion in Section 2 that $D_{(\mathbf{w}, \theta)} H\left(0, \mathbf{0}, p_{N}\right)$ is an isomorphism from $\mathbf{V} \times Q$ onto $\mathbf{V}^{*} \times Q^{*}$. Furthermore, one can verify that $t \mapsto H(t, \mathbf{w}, \theta)$ is weakly differentiable for every $(\mathbf{w}, \theta) \in \mathbf{V} \times Q$ and

$$
\begin{aligned}
\frac{\partial}{\partial t}\left\langle H_{1}(t, \mathbf{w}, \theta), \varphi\right\rangle= & \frac{\partial}{\partial t} a^{t}\left(\mathbf{w}+\mathbf{u}_{N}, \varphi\right)+\frac{\partial}{\partial t} b^{t}(\varphi, \theta) \\
& -\int_{\Omega} \dot{\delta}_{t} \mathbf{f}^{t} \cdot \varphi-\int_{\Omega} \delta_{t} \nabla \mathbf{f}^{T} \circ F_{t} h \cdot \varphi \\
& -\lambda \int_{\Gamma} \dot{\delta}_{t} \varphi \cdot M_{t} \nu-\lambda \int_{\Gamma} \delta_{t} \varphi \cdot \dot{M}_{t} \nu \\
\frac{\partial}{\partial t}\left\langle H_{2}(t, \mathbf{w}, \theta), \chi\right\rangle= & \dot{b}^{t}\left(\mathbf{w}+\mathbf{u}_{N}, \chi\right) .
\end{aligned}
$$

Since the derivatives of $\delta_{t}$ and $M_{t}$ with respect to $t$ exist in $L^{\infty}(\Omega)$, respectively $L^{\infty}\left(\Omega, \mathbb{R}^{2 \times 2}\right)$ one can show that the limits defining the above weak derivatives can be taken uniformly with respect to $\varphi \in \mathbf{V}$, respectively $\chi \in Q$ which implies that $t \mapsto H(t, \mathbf{w}, \theta)$ is strongly continuously differentiable. Therefore $H$ satisfies the assumptions of the implicit function theorem which ensures the strong differentiability of $t \mapsto\left(\mathbf{u}_{N}^{t}, p_{N}^{t}\right)$ in a neighborhood of 0 . Its derivative at $0,\left(\dot{\mathbf{u}}_{N}, \dot{p}_{N}\right)$, is the unique solution of the system

$$
\left\langle D_{(\mathbf{w}, \theta)} H\left(0, \mathbf{0}, p_{N}\right)\left(\dot{\mathbf{u}}_{N}, \dot{p}_{N}\right),(\varphi, \chi)\right\rangle=-\left\langle\frac{\partial}{\partial t} H\left(0, \mathbf{0}, p_{N}\right),(\varphi, \chi)\right\rangle
$$

which reads

$$
\begin{aligned}
\left(\left\langle D_{(\mathbf{w}, \theta)} H_{1}\left(0, \mathbf{0}, p_{N}\right)\left(\dot{\mathbf{u}}_{N}, \dot{p}_{N}\right), \varphi\right\rangle\right. & \left.,\left\langle D_{(\mathbf{w}, \theta)} H_{2}\left(0, \mathbf{0}, p_{N}\right)\left(\dot{\mathbf{u}}_{N}, \dot{p}_{N}\right), \chi\right\rangle\right) \\
& =-\frac{\partial}{\partial t}\left(\left\langle H_{1}\left(0, \mathbf{0}, p_{N}\right), \varphi\right\rangle,\left\langle H_{2}\left(0, \mathbf{0}, p_{N}\right), \chi\right\rangle\right)
\end{aligned}
$$

which by (4.4) is equivalent to

$$
\begin{aligned}
a\left(\dot{\mathbf{u}}_{N}, \varphi\right)+b\left(\varphi, \dot{p}_{N}\right) & =-\frac{\partial}{\partial t}\left\langle H_{1}\left(0, \mathbf{0}, p_{N}\right), \varphi\right\rangle \\
b\left(\dot{\mathbf{u}}_{N}, \chi\right) & =-\frac{\partial}{\partial t}\left\langle H_{2}\left(0, \mathbf{0}, p_{N}\right), \chi\right\rangle .
\end{aligned}
$$

One can verify

$$
\frac{\partial}{\partial t}\left\langle H_{2}\left(0, \mathbf{0}, p_{N}\right), \chi\right\rangle=\dot{b}\left(\mathbf{u}_{N}, \chi\right)
$$

similarly

$$
\begin{aligned}
\frac{\partial}{\partial t}\left\langle H_{1}\left(0, \mathbf{0}, p_{N}\right), \varphi\right\rangle= & \dot{a}\left(\mathbf{u}_{N}, \varphi\right)+\dot{b}\left(\varphi, p_{N}\right)-\int_{\Omega}\left(\operatorname{div} \mathbf{h} \mathbf{f} \cdot \varphi+\left(\nabla \mathbf{f}^{T}\right) \mathbf{h} \cdot \varphi\right) \\
& -\lambda \int_{\Gamma}(\operatorname{div} \mathbf{h} \varphi \cdot \nu-\varphi \cdot(\nabla \mathbf{h}) \nu) .
\end{aligned}
$$


For $\varphi \in \mathbf{V} \cap \mathbf{H}^{2}(\Omega)$ one can use Lemma 3.6 and (3.7), (3.3) and (3.5) to simplify the last term:

$$
\begin{aligned}
\int_{\Gamma}((\operatorname{div} \mathbf{h}) \varphi \cdot \nu-\varphi \cdot \nabla \mathbf{h} \nu) & =\int_{\Gamma}((\operatorname{div} \mathbf{h}-\nabla \mathbf{h} \nu \cdot \nu) \varphi \cdot \nu-(\varphi \cdot \tau) \nabla \mathbf{h} \nu \cdot \tau) \\
& =\int_{\Gamma}\left(\varphi \cdot \nu \operatorname{div}_{\Gamma} \mathbf{h}+\int_{\Gamma}(\nabla(\varphi \cdot \tau) \cdot \tau) \mathbf{h} \cdot \nu\right. \\
& =\int_{\Gamma}(\kappa \varphi \cdot \nu+\nabla \varphi \tau \cdot \tau+\nabla \tau \varphi \cdot \tau) \mathbf{h} \cdot \nu \\
& =\int_{\Gamma}(\kappa \varphi \cdot \nu+\nabla \varphi \tau \cdot \tau+(\nabla \tau \nu \cdot \tau)(\varphi \cdot \nu)) \mathbf{h} \cdot \nu \\
& =\int_{\Gamma}(\nabla \varphi \tau \cdot \tau) \mathbf{h} \cdot \nu
\end{aligned}
$$

which completes the proof of the theorem.

\subsection{Differentiability of $t \mapsto\left(\mathrm{u}_{D}^{t}, p_{D}^{t}\right)$}

The analysis of the differentiability of $t \mapsto\left(\mathbf{u}_{D}^{t}, p_{D}^{t}\right)$ is more difficult since the family of perturbed problems $(1.2)_{t}$ is well posed only in the space $V_{0, \Gamma_{t}} \times Q_{0, t}$. However, the transported solutions $\left(\mathbf{u}_{D}^{t}, p_{D}^{t}\right)$ do not belong to the subspace $\mathbf{V}_{0, \Gamma} \times Q_{0}$. This is a significant difference to the discussion in the preceding section. At first we note that the condition $\int_{\Omega_{t}} p_{D, t}=0$ leads to $\int_{\Omega} \delta_{t} p_{D}^{t}=0$. Secondly we note that by (3.2) the condition $\mathbf{u}_{D, t} \cdot \nu_{t}=0$ on $\Gamma_{t}$ is equivalent to

$$
\left(\mathbf{u}_{D, t} \cdot \nu_{t}\right) \circ F_{t}=\mathbf{u}_{D}^{t} \cdot \nu^{t}=\mathbf{u}_{D}^{t} \cdot \frac{M_{t} \nu}{\left|M_{t} \nu\right|}=0 \quad \text { on } \Gamma .
$$

Hence $\mathbf{u}_{D}^{t}$ satisfies on $\Gamma$ the boundary condition

$$
M_{t}^{T} \mathbf{u}_{D}^{t} \cdot \nu=0 \quad \text { on } \Gamma
$$

and therefore does not belong to $\mathbf{V}_{0, \Gamma}$. Transforming $(1.2)_{t}$ to the reference domain we obtain

Lemma 4.3. For each $t \in I$ the pair $\left(\mathbf{u}_{D}^{t}, p_{D}^{t}\right)$ is the unique solution of the following problem:

$$
\begin{cases}\text { Find }\left(\mathbf{u}_{D}^{t}, p_{D}^{t}\right) \in \mathbf{H}^{1}(\Omega) \times Q_{0}^{t}, \text { such that: } & \\ a^{t}\left(\mathbf{u}_{D}^{t}, \varphi^{t}\right)+b^{t}\left(\varphi^{t}, p_{D}^{t}\right)=\int_{\Omega} \delta_{t} \mathbf{f}^{t} \cdot \varphi^{t} & \forall \varphi^{t} \in \mathbf{V}_{0}^{t}, \\ b^{t}\left(\mathbf{u}_{D}^{t}, \chi^{t}\right)=0, & \forall \chi^{t} \in Q_{0}^{t}, \\ \mathbf{u}_{D}^{t}=\mathbf{g} & \text { on } \Gamma_{f}, \\ \mathbf{u}_{D}^{t} \cdot M_{t} \nu=0 & \text { on } \Gamma,\end{cases}
$$

where

$$
\begin{aligned}
& \mathbf{V}_{0}^{t}=\left\{\varphi \in \mathbf{V}: M_{t}^{T} \varphi \cdot \nu=0 \text { on } \Gamma\right\} \\
& Q_{0}^{t}=\left\{\chi \in Q: \int_{\Omega} \delta_{t} \chi=0\right\} .
\end{aligned}
$$

The boundary condition in $\mathbf{V}_{0}^{t}$ suggests to consider

$$
\mathbf{w}^{t}=M_{t}^{T}\left(\mathbf{u}_{D}^{t}-\mathbf{u}_{D}^{\star}\right),
$$


where $\left(\mathbf{u}_{D}^{\star}, p_{D}^{\star}\right)$ is the solution of the following problem

$$
\begin{cases}-\operatorname{div} \sigma\left(\mathbf{u}_{D}^{\star}\right)+\nabla p_{D}^{\star}=0 & \text { in } \Omega, \\ \operatorname{div} \mathbf{u}_{D}^{\star}=0 & \text { in } \Omega, \\ \mathbf{u}_{D}^{\star}=\mathbf{g} & \text { on } \Gamma_{f}, \\ \mathbf{u}_{D}^{\star}=0 & \text { on } \Gamma .\end{cases}
$$

Observe that $\mathbf{w}^{t} \in \mathbf{V}_{0}$ holds for all $t \in I$. This motivates to introduce the following family of auxiliary problems in $\mathbf{V}_{0} \times Q_{0}$ : For each $t \in I$

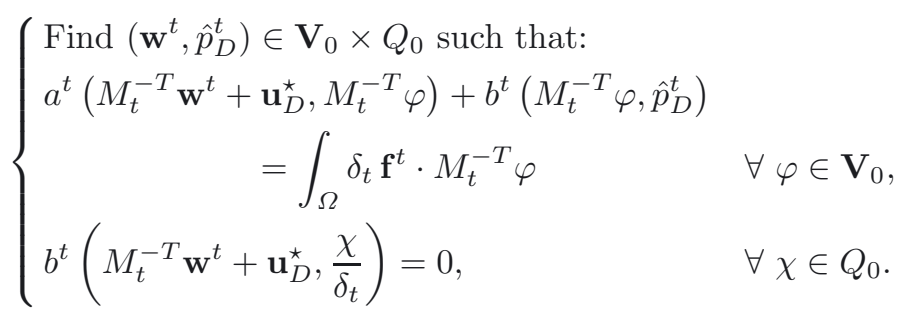

Problems (4.5) and (4.7) are related as follows:

Lemma 4.4. Let $\left(\mathbf{w}^{t}, \hat{p}_{D}^{t}\right) \in \mathbf{V}_{0} \times Q_{0}$ be a solution of (4.7) and define

$$
\left(\mathbf{u}_{D}^{t}, p_{D}^{t}\right)=\left(M_{t}^{-T} \mathbf{w}^{t}+\mathbf{u}_{D}^{\star}, \hat{p}_{D}^{t}+c(t)\right)
$$

with

$$
c(t)=-\frac{\int_{\Omega} \delta_{t} \hat{p}_{D}^{t}}{\int_{\Omega} \delta_{t}}
$$

then $\left(\mathbf{u}_{D}^{t}, p_{D}^{t}\right) \in \mathbf{H}^{1}(\Omega) \times Q_{0}^{t}$ is the solution of $(4.5)$. Conversely if $\left(\mathbf{u}_{D}^{t}, p_{D}^{t}\right) \in \mathbf{H}^{1}(\Omega) \times Q_{0}^{t}$ is the unique solution of (4.5) then

$$
\left(\mathbf{w}^{t}, \hat{p}_{D}^{t}\right)=\left(M_{t}^{T}\left(\mathbf{u}_{D}^{t}-\mathbf{u}_{D}^{\star}\right), p_{D}^{t}-\frac{1}{|\Omega|} \int_{\Omega} p_{D}^{t}\right)
$$

is a solution of (4.7)

As a consequence we conclude that (4.7) admits a unique solution for every $t \in I$.

Lemma 4.5. The mapping $t \mapsto\left(\mathbf{w}^{t}, \hat{p}_{D}^{t}\right)$ is continuously differentiable in a neighborhood of 0 and we have that $\left(\dot{\mathbf{w}}, \dot{\hat{p}}_{D}\right)=\left.\frac{\partial}{\partial t}\left(\mathbf{w}^{t}, \hat{p}_{D}^{t}\right)\right|_{t=0} \in \mathbf{V}_{0} \times Q_{0}$ is the solution of the following variational problem:

$$
\begin{cases}\text { Find }\left(\dot{\mathbf{w}}, \dot{\hat{p}}_{D}\right) \in \mathbf{V}_{0} \times Q_{0} \text { such that: } & \\ a(\dot{\mathbf{w}}, \varphi)+b\left(\varphi, \dot{\hat{p}}_{D}\right)=\hat{L}_{D}(\varphi) & \forall \varphi \in \mathbf{V}_{0}, \\ b(\dot{\mathbf{w}}, \chi)=-b\left(\nabla h^{T}\left(\mathbf{u}_{D}-\mathbf{u}_{D}^{\star}\right), \chi\right)-\dot{b}\left(\mathbf{u}_{D}, \chi\right) & \forall \chi \in Q_{0}\end{cases}
$$

where

$$
\begin{aligned}
\hat{L}_{D}(\varphi)= & -a\left(\nabla \mathbf{h}^{T}\left(\mathbf{u}_{D}-\mathbf{u}_{D}^{\star}\right), \varphi\right)-a\left(\mathbf{u}_{D}, \nabla \mathbf{h}^{T} \varphi\right)-\dot{a}\left(\mathbf{u}_{D}, \varphi\right) \\
& -b\left(\nabla \mathbf{h}^{T} \varphi, p_{D}\right)-\dot{b}\left(\varphi, p_{D}\right)+\int_{\Omega} \operatorname{div} \mathbf{h} \mathbf{f} \cdot \varphi \\
& +\int_{\Omega} \nabla \mathbf{f}^{T} \mathbf{h} \cdot \varphi+\int_{\Omega} \mathbf{f} \cdot \nabla \mathbf{h}^{T} \varphi
\end{aligned}
$$


Proof. Defining the function $H: I \times \mathbf{V}_{0} \times Q_{0} \rightarrow \mathbf{V}_{0}^{*} \times Q_{0}^{*}$ by

$$
\begin{aligned}
\left\langle H_{1}(t, \mathbf{w}, \theta), \varphi\right\rangle= & a^{t}\left(M_{t}^{-T} \mathbf{w}+\mathbf{u}_{D}^{\star}, M_{t}^{-T} \varphi\right) & & \\
& +b^{t}\left(M_{t}^{-T} \varphi, \theta\right)-\int_{\Omega} \delta_{t} \mathbf{f}^{t} \cdot M_{t}^{-T} \varphi & & \forall \varphi \in \mathbf{V}_{0}, \\
\left\langle H_{2}(t, \mathbf{w}, \theta), \chi\right\rangle= & b^{t}\left(M_{t}^{-T} \mathbf{w}+\mathbf{u}_{D}^{\star}, \frac{\chi}{\delta_{t}}\right) & & \forall \chi \in Q_{0},
\end{aligned}
$$

we note that (4.7) is equivalent to:

$$
\left\langle H\left(t, \mathbf{w}^{t}, \hat{p}_{D}^{t}\right),(\varphi, \chi)\right\rangle=0, \quad(\varphi, \chi) \in \mathbf{V}_{0} \times Q_{0} .
$$

Arguing as in the proof of Theorem 4.2 we find that $t \rightarrow\left(\mathbf{w}^{t}, \hat{p}_{D}^{t}\right)$ is continuously differentiable in a neighborhood of 0 and that $\left(\dot{\mathbf{w}}^{t}, \dot{\hat{p}}_{D}^{t}\right)=\left.\frac{\partial}{\partial t}\left(\mathbf{w}^{t}, \hat{p}_{D}^{t}\right)\right|_{t=0}$ satisfies:

$$
\begin{cases}a(\dot{\mathbf{w}}, \varphi)+b\left(\varphi, \dot{\hat{p}}_{D}^{t}\right)=-\frac{\partial}{\partial t}\left\langle H_{1}\left(0, \mathbf{w}^{0}, \hat{p}_{D}^{0}\right), \varphi\right\rangle & \forall \varphi \in \mathbf{V}_{0}, \\ b(\dot{\mathbf{w}}, \chi)=-\frac{\partial}{\partial t}\left\langle H_{2}\left(0, \mathbf{w}^{0}, \hat{p}_{D}^{0}\right), \chi\right\rangle, & \forall \chi \in Q_{0} .\end{cases}
$$

Using (3.9) and (3.10) and the definition of the bilinear forms $\dot{a}(\mathbf{u}, \mathbf{v})$ and $\dot{b}(u, v)$, and observing

$$
\mathbf{w}^{0}+\mathbf{u}_{D}^{0}=\mathbf{u}_{D}, \quad \hat{p}_{D}^{0}=p_{D}
$$

we obtain after some calculations:

$$
-\frac{\partial}{\partial t}\left\langle H_{1}\left(0, \mathbf{w}^{0}, \hat{p}_{D}^{0}\right), \varphi\right\rangle=\hat{L}_{D}(\varphi)
$$

Similarly one finds:

$$
-\frac{\partial}{\partial t}\left\langle H_{2}\left(0, \mathbf{w}^{0}, \hat{p}_{D}^{0}\right), \chi\right\rangle=\dot{b}\left(\mathbf{u}_{D}, \chi\right)+b\left(\nabla \mathbf{h}^{T}\left(\mathbf{u}_{D}-\mathbf{u}_{D}^{\star}\right), \chi\right)-b\left(\mathbf{u}_{D}, \operatorname{div} \mathbf{h} \chi\right), \quad \chi \in Q_{0} .
$$

The last term however vanishes since $\operatorname{div} \mathbf{u}_{D}=0$.

The differentiability of $t \mapsto\left(\mathbf{w}^{t}, \hat{p}_{D}^{t}\right)$ then entails the differentiability of $t \mapsto\left(\mathbf{u}_{D}^{t}, p_{D}^{t}\right)$ and $c \mapsto c(t)$.

Theorem 4.6. The mapping $t \mapsto\left(\mathbf{u}_{D}^{t}, p_{D}^{t}\right)$ is continuously differentiable in a neighborhood of 0 with respect to the topology of $\mathbf{V} \times Q$. At $t=0$ its derivative $\left(\dot{\mathbf{u}}_{D}, \dot{p}_{D}\right) \in \mathbf{V} \times Q$ is the solution of the following variational problem:

$$
\begin{cases}\text { Find }\left(\dot{\mathbf{u}}_{D}, \dot{p}_{D}\right) \in \mathbf{V} \times Q \text { such that: } & \\ a\left(\dot{\mathbf{u}}_{D}, \varphi\right)+b\left(\varphi, \dot{p}_{D}\right)=L_{D}(\varphi) & \forall \varphi \in \mathbf{V}_{0}, \\ b\left(\dot{\mathbf{u}}_{D}, \chi\right)=-\dot{b}\left(\mathbf{u}_{D}, \chi\right) & \forall \chi \in Q_{0}, \\ \dot{\mathbf{u}}_{D} \cdot \nu=\left(\nabla \mathbf{h}^{T}\right) \mathbf{u}_{D} \cdot \nu & \text { on } \Gamma,\end{cases}
$$

where

$$
\begin{aligned}
L_{D}(\varphi):= & -\dot{a}\left(\mathbf{u}_{D}, \varphi\right)-\dot{b}\left(\varphi, p_{D}\right)+\int_{\Omega} \operatorname{div} \mathbf{h} \mathbf{f} \cdot \varphi+\int_{\Omega} \nabla \mathbf{f}^{T} \mathbf{h} \cdot \varphi \\
& -\int_{\Gamma}\left(2 \nu^{T} \sigma\left(\mathbf{u}_{D}\right) \nu-p_{D}\right)\left(\nabla \mathbf{h}^{T} \tau \cdot \nu\right)(\varphi \cdot \tau)
\end{aligned}
$$


Proof. By Lemma 4.4 we have

$$
\left(\mathbf{u}_{D}^{t}, p_{D}^{t}\right)=\left(M_{t}^{-T} \mathbf{w}^{t}+\mathbf{u}_{D}^{\star}, \hat{p}_{D}^{t}+c(t)\right) .
$$

Then the differentiability of $t \mapsto\left(\mathbf{u}_{D}^{t}, p_{D}^{t}\right)$ follows from Lemma 4.5. In particular we obtain

$$
\left(\dot{\mathbf{u}}_{D}, \dot{p}_{D}\right)=\left(\nabla \mathbf{h}^{T}\left(\mathbf{u}_{D}-\mathbf{u}_{D}^{\star}\right)+\dot{\mathbf{w}}, \dot{\hat{p}}_{D}+\dot{c}(0)\right) .
$$

Inserting this expression into (4.8) results in:

$$
\begin{aligned}
a\left(\dot{\mathbf{u}}_{D}, \varphi\right) & +b\left(\varphi, \dot{p}_{D}\right)=\hat{L}_{D}(\varphi)+a\left(\nabla \mathbf{h}^{T}\left(\mathbf{u}_{D}-\mathbf{u}_{D}^{\star}\right), \varphi\right)+b(\varphi, \dot{c}(0)), \quad \varphi \in \mathbf{V}_{0} \\
b\left(\dot{\mathbf{u}}_{D}, \chi\right) & =b\left(\nabla \mathbf{h}^{T}\left(\mathbf{u}_{D}-\mathbf{u}_{D}^{\star}\right), \chi\right)+b(\dot{\mathbf{w}}, \chi) \\
& =-\dot{b}\left(\mathbf{u}_{D}, \chi\right)=-\int_{\Omega} \chi \nabla \mathbf{h}^{T}: \nabla \mathbf{u}_{D}, \quad \chi \in Q_{0} .
\end{aligned}
$$

Next observe that $\varphi \in \mathbf{V}_{0}$ implies

$$
b(\varphi, \dot{c}(0))=\dot{c}(0) \int_{\Omega} \operatorname{div} \varphi=\dot{c}(0) \int_{\partial \Omega} \varphi \cdot \nu=0 .
$$

Furthermore one finds

$$
\begin{aligned}
\hat{L}_{D}(\varphi) & +a\left(\nabla \mathbf{h}^{T}\left(\mathbf{u}_{D}-\mathbf{u}_{D}^{\star}\right), \varphi\right)=-a\left(\mathbf{u}_{D}, \nabla \mathbf{h}^{T} \varphi\right)-b\left(\nabla \mathbf{h}^{T} \varphi, p_{D}\right) \\
& -\dot{a}\left(\mathbf{u}_{D}, \varphi\right)-\dot{b}\left(\varphi, p_{D}\right)+\int_{\Omega} \operatorname{div} \mathbf{h} \mathbf{f} \cdot \varphi+\int_{\Omega} \nabla \mathbf{f}^{T} \mathbf{h} \cdot \varphi+\int_{\Omega} \mathbf{f} \cdot \nabla \mathbf{h}^{T} \varphi .
\end{aligned}
$$

We have, using (1.2)

$$
a\left(\mathbf{u}_{D}, \nabla \mathbf{h}^{T} \varphi\right)+b\left(\nabla \mathbf{h}^{T} \varphi, p_{D}\right)=\int_{\Omega} \mathbf{f} \cdot \nabla \mathbf{h}^{T} \varphi+\int_{\Gamma}\left(2 \sigma\left(\mathbf{u}_{D}\right) \nu-p_{D} \nu\right) \cdot \nabla \mathbf{h}^{T} \varphi .
$$

Decomposing successively $2 \sigma\left(\mathbf{u}_{D}\right) \nu-p_{D} \nu$ and $\varphi$ into its tangential and normal components one can verify using the boundary condition for $\mathbf{u}_{D}$ and $\varphi \cdot \nu=0$ on $\Gamma$

$$
\int_{\Gamma}\left(2 \sigma\left(\mathbf{u}_{D}\right) \nu-p_{D} \nu\right) \cdot \nabla \mathbf{h}^{T} \varphi=\int_{\Gamma}\left(2 \sigma\left(\mathbf{u}_{D}\right) \nu \cdot \nu-p_{D}\right)(\varphi \cdot \tau) \nabla \mathbf{h}^{T} \tau \cdot \nu
$$

which inserted into $\hat{L}_{D}(\varphi)+a\left(\nabla \mathbf{h}^{T}\left(\mathbf{u}_{D}-\mathbf{u}_{D}^{\star}\right), \varphi\right)$ leads to the expression given for $L_{D}(\varphi)$.

The weak form of the boundary condition for $\mathbf{u}_{D, t}$ on $\Gamma$ can be expressed as

$$
\int_{\Gamma} \delta_{t} \chi \mathbf{u}_{D}^{t} \cdot M_{t} \nu=0, \quad \chi \in L^{2}(\Gamma), t \in I
$$

Differentiating this identity at $t=0$ results in

$$
\int_{\Gamma} \operatorname{div} \mathbf{h} \chi \mathbf{u}_{D} \cdot \nu+\int_{\Gamma} \chi \dot{\mathbf{u}}_{D} \cdot \nu-\int_{\Gamma} \chi \mathbf{u}_{D} \cdot \nabla \mathbf{h} \nu=0
$$

which implies the boundary condition for $\dot{\mathbf{u}}_{D}$ on $\Gamma$

$$
\dot{\mathbf{u}}_{D} \cdot \nu=\nabla \mathbf{h}^{T} \mathbf{u}_{D} \cdot \nu
$$




\section{The Differentiability of $t \mapsto J\left(\Omega_{t}\right)$}

In this section we analyze the Eulerian derivative of $J$ at $\Omega$ in a direction $\mathbf{h} \in \mathcal{S}$. For this purpose we shall need the following two auxiliary results.

Lemma 5.1. The following equalities hold on $\Gamma$

$$
\begin{aligned}
& \sigma\left(\mathbf{u}_{D}\right): \sigma\left(\mathbf{u}_{D}\right)=2\left(\nu^{T} \sigma\left(\mathbf{u}_{D}\right) \nu\right)^{2}, \\
& \sigma\left(\mathbf{u}_{N}\right): \sigma\left(\mathbf{u}_{N}\right)=\frac{1}{2}\left(p_{N}+\lambda\right)^{2} .
\end{aligned}
$$

Proof. Let $\mathbf{u} \in \mathbf{V}$ denote either $\mathbf{u}_{N}$ or $\mathbf{u}_{D}$. Then

$$
\operatorname{tr} \sigma(\mathbf{u})=\operatorname{div} \mathbf{u}=0
$$

implies

$$
\nu^{T} \sigma(\mathbf{u}) \nu=-\tau^{T} \sigma(\mathbf{u}) \tau
$$

Defining the orthogonal matrix $O=(\nu, \tau)$ the boundary condition $\nu^{T} \sigma(\mathbf{u}) \tau=0$ on $\Gamma$ implies

$$
O^{T} \sigma(\mathbf{u}) O=\left(\begin{array}{cc}
\nu^{T} \sigma(\mathbf{u}) \nu & \nu^{T} \sigma(\mathbf{u}) \tau \\
-\tau^{T} \sigma(\mathbf{u}) \nu & \tau^{T} \sigma(\mathbf{u}) \tau
\end{array}\right)=\nu^{T} \sigma(\mathbf{u}) \nu\left(\begin{array}{rr}
1 & 0 \\
0 & -1
\end{array}\right)
$$

and therefore

$$
\sigma(\mathbf{u})=\nu^{T} \sigma(\mathbf{u}) \nu O\left(\begin{array}{rr}
1 & 0 \\
0 & -1
\end{array}\right) O^{T}
$$

This entails

$$
\sigma(\mathbf{u}): \sigma(\mathbf{u})=\operatorname{tr}(\sigma(\mathbf{u}) \sigma(\mathbf{u}))=2\left(\nu^{T} \sigma(\mathbf{u}) \nu\right)^{2} .
$$

Substituting $\mathbf{u}_{N}$, respectively $\mathbf{u}_{D}$ for $\mathbf{u}$ yields the desired result.

Lemma 5.2. For $A=A^{T} \in H^{1}\left(\Omega, \mathbb{R}^{2 \times 2}\right), \mathbf{h} \in \mathcal{S}$ and $\varphi \in \mathbf{H}^{2}(\Omega)$ we have

$$
\int_{\Omega} A: \nabla \mathbf{h} \nabla \varphi=\int_{\Gamma} \nabla \varphi^{T} \mathbf{h} \cdot A \nu-\int_{\Omega} \operatorname{div} A \cdot \nabla \varphi^{T} \mathbf{h}-\int_{\Omega}((\mathbf{h} \cdot \nabla) \sigma(\varphi)): A .
$$

Proof. This identity can be verified by integration by parts.

Theorem 5.3. The functional

$$
J\left(\Omega_{t}\right)=2 \int_{\Omega_{t}}\left|\sigma\left(\mathbf{u}_{D, t}-\mathbf{u}_{N, t}\right)\right|^{2}
$$

is shape differentiable at $\Omega$. For $\mathbf{h} \in \mathcal{S}$ the Eulerian derivative is given by

$$
\begin{aligned}
J^{\prime}(\Omega, \mathbf{h})= & 2 \int_{\Gamma}\left(\mathbf{f} \cdot\left(\mathbf{u}_{N}-\mathbf{u}_{D}\right)+\left(\nabla\left(p_{D}-2 \nu^{T} \sigma\left(\mathbf{u}_{D}\right) \nu\right)\right) \cdot \mathbf{u}_{D}\right. \\
& \left.+\left(\left|\sigma\left(\mathbf{u}_{D}\right)\right|^{2}-\left|\sigma\left(\mathbf{u}_{N}\right)\right|^{2}\right)\right) \mathbf{h} \cdot \nu
\end{aligned}
$$

Alternatively, $J^{\prime}(\Omega ; \mathbf{h})$ can be represented as

$$
\begin{aligned}
J^{\prime}(\Omega ; \mathbf{h})= & 2 \int_{\Gamma}\left(\mathbf{f} \cdot \mathbf{u}_{N}+\left(\Delta \mathbf{u}_{D}-2 \nabla\left(\nu^{T} \sigma\left(\mathbf{u}_{D}\right) \nu\right)\right) \cdot \mathbf{u}_{D}\right. \\
& \left.+2\left(\nu^{T} \sigma\left(\mathbf{u}_{D}\right) \nu\right)^{2}-\frac{1}{2}\left(\lambda+p_{N}\right)^{2}\right) \mathbf{h} \cdot \nu .
\end{aligned}
$$


Proof. We now use the argument mentioned in the introduction: if $\left(\mathbf{u}_{N}, p_{N}\right)$ is the unique solution of (1.3) corresponding to $\lambda$ then $\left(\mathbf{u}_{N}, p_{N}-\alpha\right)$ is the unique solution of (1.3) corresponding to the boundary condition $\lambda+\alpha, \alpha \in \mathbb{R}$. Therefore, replacing $\lambda$ by a suitable value $\bar{\lambda}$ we may assume without loss of generality that the corresponding solution $\left(\mathbf{u}_{N}, \bar{p}_{N}\right)$ of $(1.3)$ with $\lambda$ replaced by $\bar{\lambda}$ satisfies $\bar{p}_{N} \in Q_{0}$. Furthermore, note

$$
\bar{p}_{N}+\bar{\lambda}=p_{N}+\lambda .
$$

Transforming the cost functional (5.1) to the reference domain we obtain

$$
J\left(\Omega_{t}\right)=a^{t}\left(\mathbf{u}_{D}^{t}-\mathbf{u}_{N}^{t}, \mathbf{u}_{D}^{t}-\mathbf{u}_{N}^{t}\right)
$$

and hence by Theorems 4.2 and 4.6

$$
\begin{aligned}
J^{\prime}(\Omega ; \mathbf{h}) & =\left.\frac{\mathrm{d}}{\mathrm{d} t} J\left(\Omega_{t}\right)\right|_{t=0}=2 a\left(\dot{\mathbf{u}}_{D}-\dot{\mathbf{u}}_{N}, \mathbf{u}_{D}-\mathbf{u}_{N}\right)+\dot{a}\left(\mathbf{u}_{D}-\mathbf{u}_{N}, \mathbf{u}_{D}-\mathbf{u}_{N}\right) \\
& =: S_{1}+S_{2}
\end{aligned}
$$

where

$$
\begin{aligned}
& S_{1}=2 a\left(\dot{\mathbf{u}}_{N}, \mathbf{u}_{N}-\mathbf{u}_{D}\right)+\dot{a}\left(\mathbf{u}_{D}-\mathbf{u}_{N}, \mathbf{u}_{D}-\mathbf{u}_{N}\right) \\
& S_{2}=2 a\left(\dot{\mathbf{u}}_{D}, \mathbf{u}_{D}-\mathbf{u}_{N}\right) .
\end{aligned}
$$

Using (4.2) we obtain

$$
\begin{aligned}
S_{1}= & 2 L_{N}\left(\mathbf{u}_{N}-\mathbf{u}_{D}\right)+\dot{a}\left(\mathbf{u}_{D}-\mathbf{u}_{N}, \mathbf{u}_{D}-\mathbf{u}_{N}\right) \\
= & -2 \dot{a}\left(\mathbf{u}_{N}, \mathbf{u}_{N}-\mathbf{u}_{D}\right)+\dot{a}\left(\mathbf{u}_{D}-\mathbf{u}_{N}, \mathbf{u}_{D}-\mathbf{u}_{N}\right)-2 \dot{b}\left(\mathbf{u}_{N}-\mathbf{u}_{D}, \bar{p}_{N}\right) \\
& +2 \ell_{N}\left(\mathbf{u}_{N}-\mathbf{u}_{D}\right) \\
= & \dot{a}\left(\mathbf{u}_{D}, \mathbf{u}_{D}\right)-\dot{a}\left(\mathbf{u}_{N}, \mathbf{u}_{N}\right)-2 \dot{b}\left(\mathbf{u}_{N}-\mathbf{u}_{D}, \bar{p}_{N}\right)+2 \ell_{N}\left(\mathbf{u}_{N}-\mathbf{u}_{D}\right) .
\end{aligned}
$$

Integrating $S_{2}$ by parts and using (1.2) and (1.3) with $\lambda, p_{N}$ replaced by $\bar{\lambda}, \bar{p}_{N}$ respectively we obtain

$$
\begin{aligned}
S_{2} & =2 a\left(\dot{\mathbf{u}}_{D}, \mathbf{u}_{D}-\mathbf{u}_{N}\right) \\
& =-4 \int_{\Omega} \operatorname{div}\left(\sigma\left(\mathbf{u}_{D}-\mathbf{u}_{N}\right)\right) \cdot \dot{\mathbf{u}}_{D}+4 \int_{\Gamma} \nu^{T} \sigma\left(\mathbf{u}_{D}-\mathbf{u}_{N}\right) \dot{\mathbf{u}}_{D} \\
& =-2 \int_{\Omega} \nabla\left(p_{D}-\bar{p}_{N}\right) \cdot \dot{\mathbf{u}}_{D}+4 \int_{\Gamma} \nu^{T} \sigma\left(\mathbf{u}_{D}-\mathbf{u}_{N}\right) \dot{\mathbf{u}}_{D} \\
& =2 \int_{\Omega}\left(p_{D}-\bar{p}_{N}\right) \operatorname{div} \dot{\mathbf{u}}_{D}+2 \int_{\Gamma}\left(2 \sigma\left(\mathbf{u}_{D}-\mathbf{u}_{N}\right) \nu-\left(p_{D}-\bar{p}_{N}\right) \nu\right) \cdot \dot{\mathbf{u}}_{D} \\
& =2 \int_{\Omega}\left(p_{D}-\bar{p}_{N}\right) \operatorname{div} \dot{\mathbf{u}}_{D}+2 \int_{\Gamma}\left(2 \nu^{T} \sigma\left(\mathbf{u}_{D}\right) \nu-p_{D}-\bar{\lambda}\right) \dot{\mathbf{u}}_{D} \cdot \nu .
\end{aligned}
$$

The latter expression follows from the fact that

$$
\left(2 \sigma\left(\mathbf{u}_{D}-\mathbf{u}_{N}\right) \nu-\left(p_{D}-\bar{p}_{N}\right) \nu\right) \cdot \tau=0 \quad \text { on } \Gamma .
$$

From Theorem 4.6 we deduce $\operatorname{div} \dot{\mathbf{u}}_{D}=\nabla \mathbf{h}^{T}: \nabla \mathbf{u}_{D}$ weakly in $Q_{0}$ and $\dot{\mathbf{u}}_{D} \cdot \nu=\nabla \mathbf{h}^{T} \mathbf{u}_{D} \cdot \nu$ on $\Gamma$. This together with the definition of $\dot{b}$ eventually leads to

$$
\begin{aligned}
S_{2} & =2 \int_{\Omega}\left(p_{D}-\bar{p}_{N}\right) \nabla \mathbf{h}^{T}: \nabla \mathbf{u}_{D}+2 \int_{\Gamma}\left(2 \nu^{T} \sigma\left(\mathbf{u}_{D}\right) \nu-p_{D}-\bar{\lambda}\right) \nabla \mathbf{h} \nu \cdot \mathbf{u}_{D} \\
& =2 \dot{b}\left(\mathbf{u}_{D}, p_{D}-\bar{p}_{N}\right)+2 \int_{\Gamma}\left(2 \nu^{T} \sigma\left(\mathbf{u}_{D}\right) \nu-p_{D}-\bar{\lambda}\right) \nabla \mathbf{h} \nu \cdot \mathbf{u}_{D} .
\end{aligned}
$$


Summarizing we obtain the following preliminary representation for the Eulerian derivative of $J$

$$
\begin{aligned}
J^{\prime}(\Omega ; \mathbf{h})= & \dot{a}\left(\mathbf{u}_{D}, \mathbf{u}_{D}\right)+2 \dot{b}\left(\mathbf{u}_{D}, p_{D}\right)-\left(\dot{a}\left(\mathbf{u}_{N}, \mathbf{u}_{N}\right)+2 \dot{b}\left(\mathbf{u}_{N}, \bar{p}_{N}\right)\right) \\
& +2 \ell_{N}\left(\mathbf{u}_{N}-\mathbf{u}_{D}\right)+2 \int_{\Gamma}\left(2 \nu^{T} \sigma\left(\mathbf{u}_{D}\right) \nu-p_{D}-\bar{\lambda}\right) \nabla \mathbf{h} \nu \cdot \mathbf{u}_{D} .
\end{aligned}
$$

Let us compute the quantities:

$$
\begin{aligned}
& T_{N}=\dot{a}\left(\mathbf{u}_{N}, \mathbf{u}_{N}\right)+2 \dot{b}\left(\mathbf{u}_{N}, \bar{p}_{N}\right), \\
& T_{D}=\dot{a}\left(\mathbf{u}_{D}, \mathbf{u}_{D}\right)+2 \dot{b}\left(\mathbf{u}_{D}, p_{D}\right) .
\end{aligned}
$$

Applying Lemma 5.2 with $A=2 \sigma\left(\mathbf{u}_{N}\right)-\bar{p}_{N} I$ to $T_{N}$ results in

$$
\begin{aligned}
T_{N}= & -2 \int_{\Omega}\left(2 \sigma\left(\mathbf{u}_{N}\right)-\bar{p}_{N} I\right): \nabla \mathbf{h} \nabla \mathbf{u}_{N}+2 \int_{\Omega} \operatorname{div} \mathbf{h}\left|\sigma\left(\mathbf{u}_{N}\right)\right|^{2} \\
= & 2 \int_{\Omega} \operatorname{div}\left(2 \sigma\left(\mathbf{u}_{N}\right)-\bar{p}_{N} I\right) \cdot \nabla \mathbf{u}_{N}^{T} \mathbf{h}+2 \int_{\Omega}\left(2(\mathbf{h} \cdot \nabla) \sigma\left(\mathbf{u}_{N}\right)+\operatorname{div} \mathbf{h} \sigma\left(\mathbf{u}_{N}\right)\right): \sigma\left(\mathbf{u}_{N}\right) \\
& -2 \int_{\Omega}(\mathbf{h} \cdot \nabla) \sigma\left(\mathbf{u}_{N}\right): \bar{p}_{N} I-2 \int_{\Gamma} \nabla \mathbf{u}_{N}^{T} \mathbf{h} \cdot\left(2 \sigma\left(\mathbf{u}_{N}\right)-\bar{p}_{N} I\right) \nu
\end{aligned}
$$

Observe

$$
I:(\mathbf{h} \cdot \nabla) \sigma\left(\mathbf{u}_{N}\right)=(\mathbf{h} \cdot \nabla) \operatorname{div} \mathbf{u}_{N}=0
$$

and

$$
\int_{\Omega}\left(2(\mathbf{h} \cdot \nabla) \sigma\left(\mathbf{u}_{N}\right)+\operatorname{div} \mathbf{h} \sigma\left(\mathbf{u}_{N}\right)\right): \sigma\left(\mathbf{u}_{N}\right)=\int_{\Omega} \operatorname{div}\left(\mathbf{h}\left|\sigma\left(\mathbf{u}_{N}\right)\right|^{2}\right)=\int_{\Gamma}\left|\sigma\left(\mathbf{u}_{N}\right)\right|^{2} \mathbf{h} \cdot \nu
$$

and on $\Gamma$

$$
2 \sigma\left(\mathbf{u}_{N}\right) \nu-\bar{p}_{N} \nu=\bar{\lambda} \nu
$$

Therefore we obtain

$$
T_{N}=-2 \int_{\Omega} \mathbf{f} \cdot \nabla \mathbf{u}_{N}^{T} \mathbf{h}+2 \int_{\Gamma}\left|\sigma\left(\mathbf{u}_{N}\right)\right|^{2} \mathbf{h} \cdot \nu-2 \int_{\Gamma} \bar{\lambda}\left(\nu^{T} \nabla \mathbf{u}_{N} \nu\right) \mathbf{h} \cdot \nu
$$

Similarly $T_{D}$ can be expressed as

$$
T_{D}=-2 \int_{\Omega} \mathbf{f} \cdot \nabla \mathbf{u}_{D}^{T} \mathbf{h}+2 \int_{\Gamma}\left|\sigma\left(\mathbf{u}_{D}\right)\right|^{2} \mathbf{h} \cdot \nu-2 \int_{\Gamma}\left(2 \nu^{T} \sigma\left(\mathbf{u}_{D}\right) \nu-p_{D}\right)\left(\nu^{T} \nabla \mathbf{u}_{D} \nu\right) \mathbf{h} \cdot \nu .
$$

Then $J^{\prime}(\Omega ; \mathbf{h})$ can be written as

$$
\begin{aligned}
J^{\prime}(\Omega ; \mathbf{h})= & 2 \int_{\Omega} \mathbf{f} \cdot \nabla\left(\mathbf{u}_{N}-\mathbf{u}_{D}\right)^{T} \mathbf{h}+2 \int_{\Gamma}\left(\left|\sigma\left(\mathbf{u}_{D}\right)\right|^{2}-\left|\sigma\left(\mathbf{u}_{N}\right)\right|^{2}\right) \mathbf{h} \cdot \nu \\
& +2 \int_{\Gamma}\left(2 \nu^{T} \sigma\left(\mathbf{u}_{D}\right) \nu-p_{D}-\bar{\lambda}\right) \nabla \mathbf{h} \nu \cdot \mathbf{u}_{D}-\left[\left(2 \nu^{T} \sigma\left(\mathbf{u}_{D}\right) \nu-p_{D}\right)\left(\nu^{T} \nabla \mathbf{u}_{D} \nu\right)\right] \mathbf{h} \cdot \nu \\
& +2 \ell_{N}\left(\mathbf{u}_{N}-\mathbf{u}_{D}\right)+2 \int_{\Gamma} \bar{\lambda}\left(\nu^{T} \nabla \mathbf{u}_{N} \nu\right) \mathbf{h} \cdot \nu
\end{aligned}
$$

Let us consider the term

$$
R=2 \int_{\Gamma}\left(2 \nu^{T} \sigma\left(\mathbf{u}_{D}\right) \nu-p_{D}-\bar{\lambda}\right) \nabla \mathbf{h} \nu \cdot \mathbf{u}_{D}-\left[\left(2 \nu^{T} \sigma\left(\mathbf{u}_{D}\right) \nu-p_{D}\right)\left(\nu^{T} \nabla \mathbf{u}_{D} \nu\right)\right] \mathbf{h} \cdot \nu
$$


Since $\left(\mathbf{u}_{D}, p_{D}\right) \in \mathbf{H}^{3}(\Omega) \times H^{2}(\Omega)$ and $\nu$ respectively $\tau$ can be extended to an unitary vector field in $C^{1,1}(\Omega)$ by Theorem 2.1, on can verify that $f=\left(2 \nu^{T} \sigma\left(\mathbf{u}_{D}\right) \nu-p_{D}-\bar{\lambda}\right) \mathbf{u}_{D} \cdot \tau \in H^{2}(\Omega)$.

Since

$$
\nabla \mathbf{h} \nu \cdot \mathbf{u}_{D}=(\nabla \mathbf{h} \nu \cdot \tau)\left(\mathbf{u}_{D} \cdot \tau\right)
$$

one can apply Lemma 3.6 with $f=\left(2 \nu^{T} \sigma\left(\mathbf{u}_{D}\right) \nu-p_{D}-\bar{\lambda}\right) \mathbf{u}_{D} \cdot \tau$ to obtain

$$
\begin{aligned}
\int_{\Gamma} & \left(2 \nu^{T} \sigma\left(\mathbf{u}_{D}\right) \nu-p_{D}-\bar{\lambda}\right) \mathbf{u}_{D} \cdot \tau \nabla \mathbf{h} \nu \cdot \tau \\
& =-\int_{\Gamma} \nabla\left[\left(2 \nu^{T} \sigma\left(\mathbf{u}_{D}\right) \nu-p_{D}-\bar{\lambda}\right) \mathbf{u}_{D} \cdot \tau\right] \cdot \tau \mathbf{h} \cdot \nu \\
& =\int_{\Gamma}\left[\nabla\left(p_{D}-2 \nu^{T} \sigma\left(\mathbf{u}_{D}\right) \nu\right) \cdot \mathbf{u}_{D}+\left(\bar{\lambda}+p_{D}-2 \nu^{T} \sigma\left(\mathbf{u}_{D}\right) \nu\right) \nabla \mathbf{u}_{D} \tau \cdot \tau\right] \mathbf{h} \cdot \nu
\end{aligned}
$$

Then

$$
\begin{aligned}
R= & 2 \int_{\Gamma}\left(\nabla\left(p_{D}-2 \nu^{T} \sigma\left(\mathbf{u}_{D}\right) \nu\right) \cdot \mathbf{u}_{D}+\left(p_{D}-2 \nu^{T} \sigma\left(\mathbf{u}_{D}\right) \nu\right)\left(\nabla \mathbf{u}_{D} \tau \cdot \tau+\nabla \mathbf{u}_{D} \nu \cdot \nu\right)\right. \\
& \left.+\bar{\lambda} \nabla \mathbf{u}_{D} \tau \cdot \tau\right) \mathbf{h} \cdot \nu
\end{aligned}
$$

In view of

$$
\nabla \mathbf{u}_{D} \tau \cdot \tau+\nabla \mathbf{u}_{D} \nu \cdot \nu=\operatorname{div} \mathbf{u}_{D}=0
$$

we arrive at

$$
R=2 \int_{\Gamma}\left(\nabla\left(p_{D}-2 \nu^{T} \sigma\left(\mathbf{u}_{D}\right) \nu\right) \cdot \mathbf{u}_{D}+\bar{\lambda} \nabla \mathbf{u}_{D} \tau \cdot \tau\right) \mathbf{h} \cdot \nu .
$$

Therefore using the expression of $\ell_{N}$ and $R$ one can easily verify that $J^{\prime}(\Omega ; \mathbf{h})$ can be written as

$$
\begin{aligned}
J^{\prime}(\Omega ; \mathbf{h})= & 2 \int_{\Omega} \mathbf{f} \cdot \nabla\left(\mathbf{u}_{N}-\mathbf{u}_{D}\right)^{T} \mathbf{h}+\left(\mathbf{f} \operatorname{div} \mathbf{h}+\nabla \mathbf{f}^{T} \mathbf{h}\right) \cdot\left(\mathbf{u}_{N}-\mathbf{u}_{D}\right) \\
& +2 \int_{\Gamma}\left(\bar{\lambda}\left(\nu^{T} \nabla\left(\mathbf{u}_{N}\right) \nu+\tau^{T} \nabla \mathbf{u}_{N} \tau\right)+\left(\left|\sigma\left(\mathbf{u}_{D}\right)\right|^{2}-\left|\sigma\left(\mathbf{u}_{N}\right)\right|^{2}\right)\right. \\
& \left.+\left(\nabla\left(p_{D}-2 \nu^{T} \sigma\left(\mathbf{u}_{D}\right) \nu\right)\right) \cdot \mathbf{u}_{D}\right) \mathbf{h} \cdot \nu .
\end{aligned}
$$

Finally observing $\nu^{T} \nabla \mathbf{u}_{N} \nu+\tau^{T} \nabla \mathbf{u}_{N} \tau=\operatorname{div} \mathbf{u}_{N}=0$ and

$$
\begin{aligned}
\int_{\Omega} \mathbf{f} \cdot \nabla\left(\mathbf{u}_{N}-\mathbf{u}_{D}\right)^{T} \mathbf{h}+\left(\mathbf{f} \operatorname{div} \mathbf{h}+\nabla \mathbf{f}^{T} \mathbf{h}\right) \cdot\left(\mathbf{u}_{N}-\mathbf{u}_{D}\right) & =\int_{\Omega} \operatorname{div}\left(\mathbf{f} \cdot\left(\mathbf{u}_{N}-\mathbf{u}_{D}\right) \mathbf{h}\right) \\
& =\int_{\Gamma} \mathbf{f} \cdot\left(\mathbf{u}_{N}-\mathbf{u}_{D}\right) \mathbf{h} \cdot \nu,
\end{aligned}
$$

we get

$$
\begin{aligned}
J^{\prime}(\Omega, \mathbf{h})= & 2 \int_{\Gamma}\left(\mathbf{f} \cdot\left(\mathbf{u}_{N}-\mathbf{u}_{D}\right)+\nabla\left(p_{D}-2 \nu^{T} \sigma\left(\mathbf{u}_{D}\right) \nu\right) \cdot \mathbf{u}_{D}\right. \\
& \left.+\left(\left|\sigma\left(\mathbf{u}_{D}\right)\right|^{2}-\left|\sigma\left(\mathbf{u}_{N}\right)\right|^{2}\right)\right) \mathbf{h} \cdot \nu
\end{aligned}
$$

For the second representation of $J^{\prime}(\Omega ; \mathbf{h})$ use $\operatorname{div} 2 \sigma\left(\mathbf{u}_{D}\right)=\Delta \mathbf{u}_{D}$ since div $\mathbf{u}_{D}=0$ and Lemma 5.1. 


\section{Conclusion}

We have analyzed the shape derivative of a cost functional for an over determined Stokes problem which measures the gap between the solutions of the associated Dirichlet and Neumann problems. We proposed a possibility to overcome the difficulty arising from the fact that applying the method of mapping to the Dirichlet problem neither preserves the slip boundary condition for the velocity nor the normalizing condition for the pressure. We established the existence of the material derivatives of the states and derived the corresponding variational problems. They alone were used in the derivation of the shape derivative of the cost functional which eventually was expressed in terms of a boundary integral. This information can be combined with a level set technique to construct an efficient numerical iterative scheme to solve the free boundary Stokes problem which is ongoing research. For further algorithmic details in the context of the Bernoulli free boundary problem we refer to $[3]$.

Acknowledgements. The authors would like to thank the anonymous referee for their constructive and detailed advice.

\section{REFERENCES}

[1] C. Amrouche and V. Girault, Decomposition of vector spaces and application to the Stokes problem in arbitrary dimension. Czechoslovak Math. J. 44 (1994) 109-140.

[2] M. Badra, F. Caubet and M. Dambrine, Detecting an obstacle immersed in a fluid by shape optimization methods. $M^{3} A S \mathbf{2 1}$ (2011) 2069-2101.

[3] A. Ben Abda, F. Bouchon, G.H. Peichl, M. Sayeh and R. Touzani, A Dirichlet-Neumann cost functional approach for the Bernoulli problem. J. Eng. Math. 81 (2013) 157-176.

[4] G.J. Beavers and D.D. Joseph, Boundary conditions of a naturally permeable wall. J. Fluid Mech. 30 (1967) $197-207$.

[5] F. Boyer and P. Fabrie, Mathematical tools for the study of the incompressible Navier-Stokes equations and related models. Springer, New York (2012).

[6] S.C. Brenner and L.R. Scott, The Mathematical Theory of Finite Element Methods. Springer, New York (1996).

[7] M.C. Delfour and J.-P. Zolésio, Structure of Shape Derivatives for Nonsmooth Domains. J. Funct. Anal. 104 (1992) 1-33.

[8] M.C. Delfour and J.-P. Zolésio, Shapes and Geometries. SIAM (2001).

[9] K. Eppler and H. Harbrecht, On a Kohn-Vogelius like formulation of free boundary problems. Comput. Optim. Appl. 52 (2012) 69-85.

[10] G.P. Galdi, An introduction to the mathematical theory of the Navier-Stokes equations. Springer Tracts in Natural Philosophy. Springer-Verlag, New York (1994).

[11] V. Girault and P.A. Raviart, Finite element methods for Navier-Stokes equations. Springer, Berlin (1980).

[12] A. Henrot and M. Pierre, Variation et Optimisation de Formes. Vol. 48 of Math. Appl. Springer (2005).

[13] R.V. Kohn and A. McKenney, Numerical implementation of a variational method for electrical impedance tomography. Inverse Problems 6 (1990) 389-414.

[14] R.V. Kohn and M. Vogelius, Determining conductivity by boundary measurements. Commun. Pure Appl. Math. 37 (1984) 289-298.

[15] J. Lamboley and M. Pierre, Structure of shape derivatives around irregular domains and applications. J. Convex Analysis 14 (2007) 807-822.

[16] P. Plotnikov and J. Sokolowski, Compressible Navier-Stokes Equations. Vol. 73 of Monogr. Mat. Springer (2012).

[17] N. Saito, On the Stokes Equation with the Leak and Slip Boundary Conditions of Friction Type: Regularity of Solutions. Publ. RIMS, Kyoto Univ. 40 (2004) 345-383.

[18] H. Saito and L.E. Scriven, Study of coating flow by the finite element method. J. Comput. Phys. 42 (1981) 53-76.

[19] D.H. Sattinger, On the free surface of a viscous fluid motion. Proc. R. Soc. London A. 349 (1976) 183-204.

[20] W.J. Silliman and L.E. Scriven, Separating flow near a static contact line: slip at a wall and shape of a free surface. J. Comput. Phys. 34 (1980) 287-313.

[21] J. Sokolowski and J.-P. Zolésio, Introduction to Shape Optimization: Shape Sensitivity Analysis. Springer (1992).

$[22]$ V.A. Solonnikov, Solvability of three-dimensional problems with a free boundary for a stationary system of Navier-Stokes equations. J. Sov. Math. 21 (1983) 427-450.

[23] V.A. Solonnikov, On some free boundary problems for the Navier-Stokes equations with moving contact points and lines. Math. Ann. 302 (1995) 743-772.

[24] V.A. Solonnikov, On the problem of a moving contact angle. Nonlinear Anal. and Cont. Mech. Papers for the 65th Birthday of James Serrin (1998) 107-137.

[25] R. Verfürth, Finite element approximation of stationary Navier-Stokes equations with slip boundary condition. Habilitationsschrift, Report No. 75, University Bochum (1986).

[26] J.-P. Zolésio, An Optimal Design Procedure for Optimal Control Support, Convex Analysis and its Application. Lect. Notes Econ. Math. Syst. 144 (1977) 207-219. 